\title{
Beyond the electric-dipole approximation: A formulation and implementation of molecular response theory for the description of absorption of electromagnetic field radiation
}

Nanna Holmgaard List, Joanna Kauczor, Trond Saue, Hans Jorgen Aagaard Jensen and

Patrick Norman

Linköping University Post Print

\section{Tweet}

N.B.: When citing this work, cite the original article.

Original Publication:

Nanna Holmgaard List, Joanna Kauczor, Trond Saue, Hans Jorgen Aagaard Jensen and Patrick Norman, Beyond the electric-dipole approximation: A formulation and implementation of molecular response theory for the description of absorption of electromagnetic field radiation, 2015, Journal of Chemical Physics, (142), 24, 244111.

http://dx.doi.org/10.1063/1.4922697

Copyright: American Institute of Physics (AIP) http://www.aip.org/

Postprint available at: Linköping University Electronic Press http://urn.kb.se/resolve?urn=urn:nbn:se:liu:diva-120463 


\section{AIP $\begin{aligned} & \text { The Journal of } \\ & \text { Chemical Physics }\end{aligned}$}

\section{Beyond the electric-dipole approximation: A formulation and implementation of molecular response theory for the description of absorption of electromagnetic field radiation}

Nanna Holmgaard List, Joanna Kauczor, Trond Saue, Hans Jørgen Aagaard Jensen, and Patrick Norman

Citation: The Journal of Chemical Physics 142, 244111 (2015); doi: 10.1063/1.4922697

View online: http://dx.doi.org/10.1063/1.4922697

View Table of Contents: http://scitation.aip.org/content/aip/journal/jcp/142/24?ver=pdfcov

Published by the AIP Publishing

\section{Articles you may be interested in}

Static electric dipole polarizabilities of $\mathrm{An} 5+/ 6+$ and $\mathrm{AnO} 2+/ 2+(\mathrm{An}=\mathrm{U}, \mathrm{Np}$, and $\mathrm{Pu})$ ions

J. Chem. Phys. 141, 234304 (2014); 10.1063/1.4903792

Transition properties from the Hermitian formulation of the coupled cluster polarization propagator

J. Chem. Phys. 141, 124109 (2014); 10.1063/1.4896056

Breakdown of the pseudopotential approximation for magnetizabilities and electric multipole moments: Test calculations for Au, AuF, and Sn n cluster (n 20)

J. Chem. Phys. 134, 204102 (2011); 10.1063/1.3591338

Higher order two- and three-body dispersion coefficients for alkali isoelectronic sequences by a variationally stable procedure

J. Chem. Phys. 134, 144110 (2011); 10.1063/1.3577967

The search for a permanent electric dipole moment-still active, still important AIP Conf. Proc. 596, 39 (2001); 10.1063/1.1426792
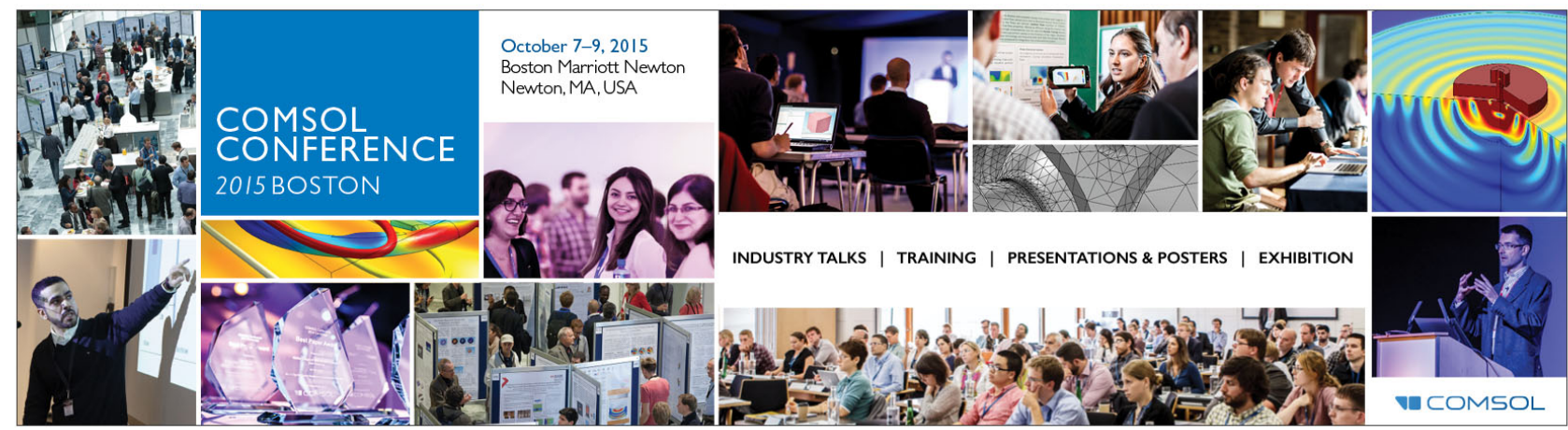


\title{
Beyond the electric-dipole approximation: A formulation and implementation of molecular response theory for the description of absorption of electromagnetic field radiation
}

\author{
Nanna Holmgaard List, ${ }^{1, a)}$ Joanna Kauczor, ${ }^{2}$ Trond Saue, ${ }^{3}$ Hans Jørgen Aagaard Jensen, ${ }^{1}$ \\ and Patrick Norman 2, b) \\ ${ }^{1}$ Department of Physics, Chemistry and Pharmacy, University of Southern Denmark, Campusvej 55, \\ DK-5230 Odense M, Denmark \\ ${ }^{2}$ Department of Physics, Chemistry and Biology, Linköping University, Linköping SE 58183, Sweden \\ ${ }^{3}$ Laboratoire de Chimie et Physique Quantiques, UMR 5626-CNRS/Université Toulouse III (Paul Sabatier), \\ 118 route de Narbonne, F-31062 Toulouse Cedex, France
}

(Received 24 March 2015; accepted 1 June 2015; published online 29 June 2015)

\begin{abstract}
We present a formulation of molecular response theory for the description of a quantum mechanical molecular system in the presence of a weak, monochromatic, linearly polarized electromagnetic field without introducing truncated multipolar expansions. The presentation focuses on a description of linear absorption by adopting the energy-loss approach in combination with the complex polarization propagator formulation of response theory. Going beyond the electric-dipole approximation is essential whenever studying electric-dipole-forbidden transitions, and in general, non-dipolar effects become increasingly important when addressing spectroscopies involving higher-energy photons. These two aspects are examined by our study of the near $K$-edge $X$-ray absorption fine structure of the alkaline earth metals $(\mathrm{Mg}, \mathrm{Ca}, \mathrm{Sr}, \mathrm{Ba}$, and $\mathrm{Ra})$ as well as the trans-polyenes. In following the series of alkaline earth metals, the sizes of non-dipolar effects are probed with respect to increasing photon energies and a detailed assessment of results is made in terms of studying the pertinent transition electron densities and in particular their spatial extension in comparison with the photon wavelength. Along the series of trans-polyenes, the sizes of non-dipolar effects are probed for X-ray spectroscopies on organic molecules with respect to the spatial extension of the chromophore. (C) 2015 AIP Publishing LLC. [http://dx.doi.org/10.1063/1.4922697]
\end{abstract}

\section{INTRODUCTION}

A large variety of spectroscopies has been developed over the years with the purpose to perform basic characterization and reach increased understanding of molecules and molecular materials in technical applications as well as biochemical contexts. ${ }^{1}$ The basic principle for all spectroscopies is that they are concerned with the interactions between external electromagnetic fields (or sometimes internal nuclear magnetic moments) and molecular charges. In many cases, the fields can be considered as weak with respect to the atomic fields and classical, such that the molecular charges represent a quantum mechanical system that is perturbed by the fields. ${ }^{2-4}$ Theory then meets and can be compared to experiment by the definition of linear and nonlinear coupling constants between the external fields and experimental observables and these coupling constants are known as response functions. ${ }^{5}$ It is clear that, while a great deal of information can be extracted from experimental spectra alone, the revelation of the detailed relationships between observed properties and molecular structures requires also the aid of first principles calculations.

To calculate the values of the response functions, it becomes necessary to introduce the given external electro-

\footnotetext{
a)Electronic mail: nhl@sdu.dk

b)Electronic mail: panor@ifm.liu.se
}

magnetic fields into the description of the quantum mechanical molecular system. This is readily achieved by means of a well known procedure referred to as the rule of minimal coupling, ${ }^{6,7}$ which introduces the auxiliary scalar and vector potentials as representations of the physical fields. This recipe is rarely ever carried out in a straightforward manner, since it, for several reasons, is highly beneficial to introduce Taylor expansions of the scalar and vector potentials about a reference point chosen to reside inside the molecular volume. Thereafter, the usual approach is to take into account spatial variations in the scalar and vector potentials to a given order in this multipole moment expansion. As simple as this strategy appears to be, a truncated expansion may introduce an unphysical and nontrivial dependence of molecular response properties on the choice of gauge origin, ${ }^{3,8,9}$ and it was only recently demonstrated that origin-independent results for spectral absorption intensities can be retrieved to arbitrary order in the propagation vector by retaining all contributing terms to the oscillator strength to the same order. ${ }^{10}$ In the vast majority of theoretical work, light-matter interactions are described within the electricdipole (ED) approximation. In the ED approximation, the wavelength of the electromagnetic radiation is assumed to be much larger than the spatial extension of the electronic transitions under consideration. Accordingly, the expanded form of the spatial part of the radiation field, $e^{i \boldsymbol{k} \cdot \boldsymbol{r}}=1+i \boldsymbol{k} \cdot \boldsymbol{r}$ $-\frac{1}{2}(\boldsymbol{k} \cdot \boldsymbol{r})^{2}+\ldots$, can be truncated at the zeroth-order term 
(unity) in $\boldsymbol{k}$, where $\boldsymbol{k}$ is the wavevector with norm $k=|\boldsymbol{k}|=\frac{\omega}{c}$ $=\frac{2 \pi}{\lambda}, \omega$ is the angular frequency, $\lambda$ the wavelength of the field, $c$ the speed of light in vacuum, and $\boldsymbol{r}$ a spatial coordinate vector. ${ }^{11}$ With the exception of transitions that for symmetry reasons are formally electric-dipole forbidden, such as $n \rightarrow \pi^{*}$ transitions in the $\mathrm{C}_{2 v}$ point group, the ED approximation will be highly accurate in the low-energy spectral region, and it should be noted that the issue of origin-dependence does not occur in the ED approximation due to the orthogonality between ground and excited states.

At high photon energies, where $k r$ exceeds unity, the spatial variations in the electromagnetic field across the molecule will become important and any finite-order multipolar expansion will eventually become questionable as the wavelength grows shorter. It is therefore natural to examine the validity of the multipolar expansions in the case of $\mathrm{X}$ ray spectroscopies. Non-dipolar effects have been studied extensively in X-ray photoelectron spectroscopy, where they are primarily manifested as deviations from dipolar angular distributions of photoelectrons, and due to its sensitivity to interferences among different photoemission channels, deviations in angle-resolved photoemission may be observed even at low photon energies (see, e.g., Refs. 12-15 and references therein). In the present work, we will consider non-dipolar effects in X-ray absorption spectroscopy (XAS) and pay particular attention to near-edge X-ray absorption fine structure (NEXAFS) spectroscopy. ${ }^{16}$ In NEXAFS, the local electronic and geometric environment at a given atomic center is probed by electronic excitations from core levels to final electronic states given in terms of the unoccupied and delocalized valence states, or the continuum. The information content of XAS relies on changes in the positions, intensities, and line widths of the resonances. For instance, the pre- $K$-edge intensity for a transition metal-ligand complex may provide insight into the covalency of the metal-ligand bond, ${ }^{17-19}$ whereas spectral features in the metal $K$ - and $L$-edge regions can be used to discriminate oxidation states and identify the coordination number of the metal atom. ${ }^{20-27}$ NEXAFS was designed for the study of the orientation of molecules chemisorbed to surfaces and has in this context been used in numerous applications. ${ }^{28-31}$ XAS can also be used to study the local atomic structure and displacement of the absorbing center in crystals ${ }^{32,33}$ and in liquid phases to, for example, detect the local hydration environment of a solute. ${ }^{34,35}$

In the present work, we conduct a critical assessment of the ED approximation in response theory calculations of linear absorption cross sections by developing the theory and implementing the code required to describe a molecular system subjected to a weak, linearly polarized electromagnetic plane wave. Since our work will not introduce any truncations whatsoever, the presented results are by construction gaugeorigin independent, and, in the language of multipolar expansions, the presented absorption spectra include contributions to infinite order. We will base our derivation of a response theory description of linear absorption on the energy-loss approach, which makes it directly linked to the formulation of resonance-convergent complex molecular properties. ${ }^{36}$ In the ED approximation, we recall that the linear absorption cross section is proportional to the imaginary part of the electric-dipole polarizability, a property which we have earlier addressed by means of the development of the complex polarization propagator (CPP) approach. ${ }^{37-39}$ In Sec. II, we will present the response functions pertinent to absorption, and in Sec. III, we detail the computationally tractable expression for the linear absorption cross section beyond the electric-dipole (BED) approximation in the framework of time-dependent density functional theory (TD-DFT).

In Sec. V, we present numerical assessments of the importance of going beyond the ED approximation in absorption spectroscopic calculations. In a first example, we study the near $K$-edge $\mathrm{X}$-ray absorption of the alkaline earth metals $(\mathrm{Mg}$, $\mathrm{Ca}, \mathrm{Sr}, \mathrm{Ba}$, and $\mathrm{Ra}$ ), which, due to the atomic symmetry, strictly separate out the electric-dipole-allowed $1 s \rightarrow n p$ transitions from all the others, and by going down the second group in the periodic table, we follow the progression of errors associated with the ED approximation as one turns from soft to hard $\mathrm{X}$-ray fields. We would like to stress that we in the present work restrict attention to a non-relativistic framework which is clearly not reliable for the heavier alkaline earth metals. On the other hand, the present study is more concerned with proof of concept than quantitative accuracy. In a second example, we study the validity of the ED approximation in applications concerned with $K$-edge spectra of extended organic molecules. For this purpose, the series of trans-polyenes has been chosen, which allows us to consider molecular systems with spatial dimensions exceeding the $1 s \rightarrow \pi^{*}$ transition wavelength.

\section{THEORY}

\section{A. The Hamiltonian}

In the presence of a time-dependent external classical electromagnetic field, a molecular system within the Born-Oppenheimer approximation can be described by the minimal-coupling form of the non-relativistic electronic Hamiltonian. ${ }^{40,41}$ In first-quantization, it reads as

$$
\begin{aligned}
\hat{H}= & \sum_{i=1}^{N}\left(\frac{1}{2 m_{e}}\left[\hat{\boldsymbol{p}}_{i}+e \boldsymbol{A}\left(\boldsymbol{r}_{i}, t\right)\right]^{2}-e \phi\left(\boldsymbol{r}_{i}, t\right)+\frac{e}{m_{e}} \boldsymbol{B}\left(\boldsymbol{r}_{i}, t\right) \cdot \hat{\boldsymbol{s}}_{i}\right) \\
& +V\left(\boldsymbol{r}_{1}, \ldots, \boldsymbol{r}_{N}\right),
\end{aligned}
$$

where the linear momentum operator is given by $\hat{\boldsymbol{p}}_{i}=-i \hbar \nabla_{i}$, $-e$ and $m_{e}$ are the charge and mass of an electron, respectively, and $N$ the total number of electrons. The second last term represents the spin Zeeman interaction, while the last term covers the nuclear-electron attraction and electron-electron repulsion operators. In the absence of sources, the electric and magnetic fields are purely transversal. Hence, within the Coulomb gauge, where the vector potential is chosen divergence free $(\nabla \cdot \boldsymbol{A}=0)^{40}$ such that $\boldsymbol{A}$ is also purely transversal, the scalar potential is constant and imposed to vanish, $\phi(\boldsymbol{r}, t)=0$. For a monochromatic, linearly polarized electromagnetic wave, the vector potential becomes

$$
\boldsymbol{A}(\boldsymbol{r}, t)=\sum_{ \pm \omega} A^{\omega} e^{-i \omega t} ; \quad \boldsymbol{A}^{ \pm \omega}= \pm \frac{\mathcal{E}_{0}}{2 i \omega} e^{ \pm i \boldsymbol{k} \cdot \boldsymbol{r}} \boldsymbol{\epsilon},
$$

where $\mathcal{E}_{0}$ is the real amplitude of the corresponding electric field [see Eq. (3)] and $\boldsymbol{k}$ is the wavevector specifying the propagation direction of the light beam. Finally, $\boldsymbol{\epsilon}$ is the 
unit vector in the direction of the polarization, which is perpendicular to the propagation direction (i.e., $\boldsymbol{\epsilon} \cdot \boldsymbol{k}=0$ ). Note that the Fourier component $\boldsymbol{A}^{\omega}$ satisfies $\boldsymbol{A}^{-\omega}=\left[\boldsymbol{A}^{\omega}\right]^{\dagger}$, which ensures the Hermiticity of the field-matter interaction operators. In the Coulomb gauge, the physical electric and magnetic fields are given by

$$
\begin{array}{ll}
\boldsymbol{E}(\boldsymbol{r}, t)=-\partial_{t} \boldsymbol{A}(\boldsymbol{r}, t)=\sum_{ \pm \omega} \boldsymbol{E}^{\omega} e^{-i \omega t} ; & \boldsymbol{E}^{\omega}=i \omega \boldsymbol{A}^{\omega}, \\
\boldsymbol{B}(\boldsymbol{r}, t)=\nabla \times \boldsymbol{A}(\boldsymbol{r}, t)=\sum_{ \pm \omega} \boldsymbol{B}^{\omega} e^{-i \omega t} ; & \boldsymbol{B}^{\omega}=i\left(\boldsymbol{k} \times \boldsymbol{A}^{\omega}\right) .
\end{array}
$$

The intensity, $I(\omega)$, of the incident electromagnetic field, as defined by the average power per unit area through a surface perpendicular to the propagation direction of the field can then be calculated from the Poynting vector, ${ }^{42}$

$$
\begin{aligned}
\boldsymbol{S}(\boldsymbol{r}, t) & =\varepsilon_{0} c^{2}(\boldsymbol{E}(\boldsymbol{r}, t) \times \boldsymbol{B}(\boldsymbol{r}, t)) \\
& =\varepsilon_{0} c^{2} \frac{\mathcal{E}_{0}^{2}}{\omega} \boldsymbol{k} \cos ^{2}(\boldsymbol{k} \cdot \boldsymbol{r}-\omega t),
\end{aligned}
$$

by performing a time-average of its magnitude over one period $T$ of the oscillation, yielding

$$
I(\omega)=\langle|S(\boldsymbol{r}, t)|\rangle_{T}=\frac{1}{T} \int_{-\frac{T}{2}}^{\frac{T}{2}}|S(\boldsymbol{r}, t)| d t=\frac{1}{2} \varepsilon_{0} c \mathcal{E}_{0}^{2} .
$$

Returning to the Hamiltonian operator, the choice of the Coulomb gauge leads to a vanishing commutator between the vector potential and the momentum operator, and thus, the Hamiltonian can be written as

$$
\begin{aligned}
\hat{H}= & \sum_{i=1}^{N}\left(\frac{1}{2 m_{e}}\left[\hat{\boldsymbol{p}}_{i}^{2}+e^{2} \boldsymbol{A}^{2}\left(\boldsymbol{r}_{i}, t\right)+2 e \boldsymbol{A}\left(\boldsymbol{r}_{i}, t\right) \cdot \hat{\boldsymbol{p}}_{i}\right]\right. \\
& \left.+\frac{e}{m_{e}} \boldsymbol{B}\left(\boldsymbol{r}_{i}, t\right) \cdot \hat{\boldsymbol{s}}_{i}\right)+V\left(\boldsymbol{r}_{1}, \ldots, \boldsymbol{r}_{N}\right) .
\end{aligned}
$$

Since we are concerned with one-photon absorption, we henceforth invoke the weak-field approximation and neglect the term quadratic in the vector potential, although this formally breaks gauge invariance. Moreover, we will focus on closed-shell molecules for which the spin Zeeman term drops out. The field-matter interaction operator, $\hat{V}^{\text {int }}$, can now be decomposed in the frequency domain by inserting the expression for the vector potential in Eq. (2),

$$
\begin{aligned}
& \hat{V}^{\text {int }}(t)=\sum_{ \pm \omega} \hat{V}^{\omega} e^{-i \omega t} ; \quad \hat{V}^{\omega}=\sum_{i=1}^{N} \hat{v}^{\omega}(i) ; \\
& \hat{v}^{ \pm \omega}=\frac{e}{m_{e}}\left(\boldsymbol{A}^{ \pm \omega} \cdot \hat{\boldsymbol{p}}\right)=\mp \frac{e \hbar \mathcal{E}_{0}}{2 \omega m_{e}} e^{ \pm i \boldsymbol{k} \cdot \boldsymbol{r}}(\boldsymbol{\epsilon} \cdot \boldsymbol{\nabla}),
\end{aligned}
$$

where $\hat{V}^{-\omega}=\left[\hat{V}^{\omega}\right]^{\dagger}$ follows from the Hermiticity of $\hat{V}^{\text {int }}$.

Before switching on the perturbation, we assume the molecule to be in the electronic ground state $|0\rangle$ of the unperturbed Hamiltonian,

$$
\hat{H}_{0}|0\rangle=E_{0}|0\rangle \text {. }
$$

When turning on the perturbation, we shall express the timedependent state $|0(t)\rangle$ in terms of the set of eigenfunctions $\{|n\rangle\}$ of the unperturbed Hamiltonian. To this end, we take an exponential ansatz for the parametrization of the wave function. ${ }^{43}$ Since we are focusing on expectation values, it suffices to consider the phase-isolated wave function, ${ }^{38}$ which then reads as

$$
|0(t)\rangle=e^{-i \hat{P}(t)}|0\rangle,
$$

where $\hat{P}(t)$ is a Hermitian operator defined in terms of the excitation, $\hat{R}_{n}^{\dagger}=|n\rangle\langle 0|$, and de-excitation, $\hat{R}_{n}=|0\rangle\langle n|$, state-transfer operators, and a set of complex wave function amplitudes $\left\{P_{n}\right\}$ as

$$
\hat{P}(t)=\sum_{n>0}\left(P_{n}(t) \hat{R}_{n}^{\dagger}+P_{n}^{*}(t) \hat{R}_{n}\right)
$$

To allow for a description of the resonant region, we follow the damped response formalism of Ref. 38, in which the timeevolution of the wave function amplitudes is determined by the Liouville equation augmented with a phenomenological damping term that accounts for finite lifetimes of the excited states. The amplitudes are then determined by requiring the equation to be fulfilled in each order of the perturbation (i.e., the field strength $\mathcal{E}_{0}$ ), and for our purposes, it suffices to consider the first-order amplitudes. For the perturbation in Eq. (8), we obtain

$$
P_{n}^{(1)}(t)=-\frac{i}{\hbar} \sum_{ \pm \omega}\left[\frac{\left\langle n\left|\hat{V}^{\omega}\right| 0\right\rangle}{\omega_{n 0}-\omega-i \gamma_{n 0}}\right] e^{-i \omega t}
$$

and

$$
P_{n}^{(1) *}(t)=\frac{i}{\hbar} \sum_{ \pm \omega}\left[\frac{\left\langle 0\left|\hat{V}^{\omega}\right| n\right\rangle}{\omega_{n 0}+\omega+i \gamma_{n 0}}\right] e^{-i \omega t},
$$

where the damping parameter $\gamma_{n 0}=\left(2 \tau_{n}\right)^{-1}$ is related to the inverse lifetime $\left(\tau_{n}\right)$ of the $n$th excited state and $\hbar \omega_{n 0}=E_{n}$ $-E_{0}$. Finally, the first-order corrections to the wave function become

$$
\begin{aligned}
& \left|0^{(1)}\right\rangle=-i \hat{P}^{(1)}(t)|0\rangle=-i \sum_{n>0}|n\rangle P_{n}^{(1)}(t), \\
& \left\langle 0^{(1)}\right|=i\langle 0| \hat{P}^{(1)}(t)=i \sum_{n>0} P_{n}^{(1) *}(t)\langle n|,
\end{aligned}
$$

where the explicit time-dependence of the wave function correction has been suppressed.

\section{B. Light absorption in molecular materials}

In this section, we briefly review the intrinsic response of matter to an electromagnetic field. We follow the energy-loss approach, ${ }^{36}$ which provides a natural link to the complexvalued molecular properties underlying the absorption process. One may note that going beyond the ED approximation introduces an explicit dependence in the interaction operator [see Eq. (8)] on the wavevector $\boldsymbol{k}$. Rotational averaging must therefore be taken into account when considering samples of freely rotating molecules, an issue that we will not address in the present work. We shall therefore be concerned with an oriented sample with number density $\mathcal{N}$ that consists of identical, non-interacting, closed-shell molecules subjected to a monochromatic, linearly polarized electromagnetic field. In this case, the light-matter interaction operator is given by Eq. (8), and the macroscopic properties of the sample is straightforwardly obtained as the number density times the molecular property, to the extent that local-field corrections can be ignored. 
The rate of absorption can be defined as the attenuation per unit time of the beam of light passing through an absorbing sample. Conservation of energy states that the change in total energy per unit volume stored in the electromagnetic field corrected for the energy flow through the volume-enclosing surface equals the rate of work done by the electromagnetic force on the sample. The rate of absorption within volume $V$ can then be written as ${ }^{42}$

$$
\frac{d W}{d t}=\int_{V} \boldsymbol{E}(\boldsymbol{r}, t) \cdot \boldsymbol{j}(\boldsymbol{r}, t) d \boldsymbol{r}
$$

where $j(r, t)$ is the current density in the sample. The work done by the field originates entirely from the electric component of the field because the magnetic force experienced by the electrons is perpendicular to their velocity vectors. We shall restrict our attention to cases where the currents are due to bound electrons and not due to conduction electrons. To remove the oscillatory behavior of the rate of absorption, Eq. (16) is time-averaged over one period of the oscillation. In the context of light-matter interactions, we are interested in relating the absorption process to the linear and non-linear properties of the sample. Particularly, by expanding the current density, $\boldsymbol{j}=\boldsymbol{j}^{(0)}+\boldsymbol{j}^{(1)}+\boldsymbol{j}^{(2)}+\cdots$, in orders of the perturbation (i.e., $\mathcal{E}_{0}$ ), the rate of absorbed energy can be expressed as a power series in the light intensity [Eq. (6)], ${ }^{36}$

$$
\left\langle\frac{d W}{d t}\right\rangle_{T}=\alpha(\omega) I+\beta(\omega) I^{2}+\gamma(\omega) I^{3}+\cdots,
$$

where $\alpha, \beta, \gamma$ are the so-called absorption coefficients (not to be confused with the standard dipole-dipole linear and higher-order polarizabilities). Note that terms of odd order in the perturbation have only periodic contributions, which vanish upon time-averaging, and thus do not contribute to light absorption. ${ }^{36}$

As will be shown below in the case of linear absorption, the rate of absorbed energy in orders of the intensity can be related to the imaginary parts of the even-order molecular response functions,

$$
\begin{aligned}
& \left\langle\int_{V} \boldsymbol{E} \cdot \boldsymbol{j}^{(1)} d \boldsymbol{r}\right\rangle_{T}=2 \omega \mathcal{N} \operatorname{Im}\left[\left\langle\left\langle\hat{V}^{-\omega} ; \hat{V}^{\omega}\right\rangle\right],\right. \\
& \left\langle\int_{V} \boldsymbol{E} \cdot \boldsymbol{j}^{(3)} d \boldsymbol{r}\right\rangle_{T}=6 \omega \mathcal{N} \operatorname{Im}\left[\left\langle\left\langle\hat{V}^{-\omega} ; \hat{V}^{\omega}, \hat{V}^{-\omega}, \hat{V}^{\omega}\right\rangle\right],\right. \\
& \left\langle\int_{V} \boldsymbol{E} \cdot \boldsymbol{j}^{(5)} d \boldsymbol{r}\right\rangle_{T}=20 \omega \mathcal{N} \operatorname{Im}\left[\left\langle\hat{V}^{-\omega} ; \hat{V}^{\omega}, \hat{V}^{-\omega}, \hat{V}^{\omega}, \hat{V}^{-\omega}, \hat{V}^{\omega}\right\rangle\right],
\end{aligned}
$$

where the linear response function $\left\langle\left\langle\hat{V}^{-\omega} ; \hat{V}^{\omega}\right\rangle\right\rangle$ is related to one-photon absorption, the cubic response function $\left\langle\left\langle\hat{V}^{-\omega} ; \hat{V}^{\omega}, \hat{V}^{-\omega}, \hat{V}^{\omega}\right\rangle\right\rangle$ to two-photon absorption an so on, due to a linearly polarized light source. We note that this situation is of relevance in, for example, $\mathrm{X}$-ray spectroscopies using a synchrotron as the source of tunable monochromatic and highly polarized radiation. ${ }^{28}$ In $K$-edge NEXAFS spectroscopy, for instance, transitions from spherically symmetric $1 s$-orbitals to empty (or virtual) orbitals are probed, and the polarized nature of radiation will reveal the directional properties of the final electronic state and thereby also the molecular orientation in ordered samples. ${ }^{30,31}$ With NEXAFS calculations in mind, we restrict our attention to linear absorption.

\section{Linear absorption of an ensemble of pure states}

To determine the rate of linear absorption by the ensemble, we need the electronic current density [cf. Eq. (16)]. The firstquantization form of the current density operator reads as ${ }^{4-46}$

$$
\hat{j}(\boldsymbol{r}, t)=-\frac{e}{2 m_{e}} \sum_{i=1}^{N}\left[\hat{\boldsymbol{p}}_{i}+e \boldsymbol{A}\left(\boldsymbol{r}_{i}, t\right), \delta\left(\boldsymbol{r}_{i}-\boldsymbol{r}\right)\right]_{+},
$$

where the spin contribution to the current (see for instance Ref. 47) vanishes as we consider closed-shell molecules. In the following, we will drop the last term (the diamagnetic current), in accordance with the weak-field approximation. The macroscopic induced current density is then obtained as $\boldsymbol{j}(\boldsymbol{r}, t)=\mathcal{N}\langle 0(t)|\hat{\boldsymbol{j}}(\boldsymbol{r}, t)| 0(t)\rangle$, which after insertion of the current density operator gives

$$
\boldsymbol{j}(\boldsymbol{r}, t)=-\mathcal{N} \sum_{i=1}^{N}\left[\frac{e}{2 m_{e}}\left\langle 0(t)\left|\hat{\boldsymbol{p}}_{i} \delta\left(\boldsymbol{r}_{i}-\boldsymbol{r}\right)+\delta\left(\boldsymbol{r}_{i}-\boldsymbol{r}\right) \hat{\boldsymbol{p}}_{i}\right| 0(t)\right\rangle\right] .
$$

For closed-shell molecules, the current density of the unperturbed density is vanishing everywhere. However, for the description of linear absorption, we are interested in the first-order perturbation-induced current density, which can be obtained by expanding the current in powers of the field strength. To first order, we have

$$
\boldsymbol{j}^{(1)}(\boldsymbol{r}, t)=-i \mathcal{N} \sum_{n>0}\left[\langle 0|\hat{\boldsymbol{j}}(\boldsymbol{r})| n\rangle P_{n}^{(1)}(t)-P_{n}^{(1) *}(t)\langle n|\hat{\boldsymbol{j}}(\boldsymbol{r})| 0\rangle\right] .
$$

Substituting this expression into Eq. (16) provides the expression for the absorbed energy density per unit time,

$$
\begin{aligned}
& \int \boldsymbol{E} \cdot \boldsymbol{j}^{(1)} d \boldsymbol{r}=\mathcal{N} \sum_{\omega_{1}} \omega_{1} \sum_{n>0}\left\{P_{n}^{(1)}(t) \int\left\langle 0\left|\boldsymbol{A}^{\omega_{1}} \cdot \hat{\boldsymbol{j}}(\boldsymbol{r})\right| n\right\rangle d \boldsymbol{r}-P_{n}^{(1) *}(t) \int\left\langle n\left|\boldsymbol{A}^{\omega_{1}} \cdot \hat{\boldsymbol{j}}(\boldsymbol{r})\right| 0\right\rangle d \boldsymbol{r}\right\} e^{-i \omega_{1} t} \\
& =i \mathcal{N} \sum_{\omega_{1}, \omega_{2}} \omega_{1} \frac{e}{2 m_{e} \hbar} \sum_{n>0}\left\{\left(\left\langle 0\left|\hat{\boldsymbol{p}} \cdot \hat{\boldsymbol{A}}^{\omega_{1}}\right| n\right\rangle+\left\langle 0\left|\hat{\boldsymbol{A}}^{\omega_{1}} \cdot \hat{\boldsymbol{p}}\right| n\right\rangle\right)\left[\frac{\left\langle n\left|\hat{V}^{\omega_{2}}\right| 0\right\rangle}{\omega_{n 0}-\omega_{2}-i \gamma_{n 0}}\right]\right. \\
& \left.+\left[\frac{\left\langle 0\left|\hat{V}^{\omega_{2}}\right| n\right\rangle}{\omega_{n 0}+\omega_{2}+i \gamma_{n 0}}\right]\left(\left\langle n\left|\hat{\boldsymbol{p}} \cdot \hat{\boldsymbol{A}}^{\omega_{1}}\right| 0\right\rangle+\left\langle n\left|\hat{\boldsymbol{A}}^{\omega_{1}} \cdot \hat{\boldsymbol{p}}\right| 0\right\rangle\right)\right\} e^{-i\left(\omega_{1}+\omega_{2}\right) t} \\
& =i \mathcal{N} \sum_{\omega_{1}, \omega_{2}} \omega_{1}\left\{\frac{1}{\hbar} \sum_{n>0}\left[\frac{\left\langle 0\left|\hat{V}^{\omega_{1}}\right| n\right\rangle\left\langle n\left|\hat{V}^{\omega_{2}}\right| 0\right\rangle}{\omega_{n 0}-\omega_{2}-i \gamma_{n 0}}+\frac{\left\langle 0\left|\hat{V}^{\omega_{2}}\right| n\right\rangle\left\langle n\left|\hat{V}^{\omega_{1}}\right| 0\right\rangle}{\omega_{n 0}+\omega_{2}+i \gamma_{n 0}}\right]\right\} e^{-i\left(\omega_{1}+\omega_{2}\right) t},
\end{aligned}
$$


where the first-order corrections to the wave function [Eqs. (14) and (15)] have been used. The third equality follows by collecting terms in the numerator and making use of the divergence free property of the vector potential. We define the linear response function

$$
\begin{aligned}
\left\langle\left\langle\hat{V}^{\omega_{1}} ; \hat{V}^{\omega_{2}}\right\rangle\right\rangle= & G\left(\omega_{1} ; \omega_{2}\right) \\
= & -\frac{1}{\hbar} \sum_{n>0}\left[\frac{\left\langle 0\left|\hat{V}^{\omega_{1}}\right| n\right\rangle\left\langle n\left|\hat{V}^{\omega_{2}}\right| 0\right\rangle}{\omega_{n 0}-\omega_{2}-i \gamma_{n 0}}\right. \\
& \left.+\frac{\left\langle 0\left|\hat{V}^{\omega_{2}}\right| n\right\rangle\langle n| \hat{V}^{\omega_{1}|0\rangle}}{\omega_{n 0}+\omega_{2}+i \gamma_{n 0}}\right],
\end{aligned}
$$

where

$$
\begin{aligned}
\operatorname{Re}\left[G\left(\omega_{1} ; \omega_{2}\right)\right]= & -\frac{1}{\hbar} \sum_{n>0}\left[\frac{\left(\omega_{n 0}-\omega_{2}\right)\left\langle 0\left|\hat{V}^{\omega_{1}}\right| n\right\rangle\left\langle n\left|\hat{V}^{\omega_{2}}\right| 0\right\rangle}{\left(\omega_{n 0}-\omega_{2}\right)^{2}+\gamma_{n 0}^{2}}\right. \\
& \left.+\frac{\left.\left(\omega_{n 0}+\omega_{2}\right)\left\langle 0\left|\hat{V}^{\omega_{2}}\right| n\right\rangle\langle n| \hat{V}^{\omega_{1}|0\rangle}\right]}{\left(\omega_{n 0}+\omega_{2}\right)^{2}+\gamma_{n 0}^{2}}\right], \quad \\
\operatorname{Im}\left[G\left(\omega_{1} ; \omega_{2}\right)\right]=- & \frac{1}{\hbar} \sum_{n>0} \gamma_{n 0}\left[\frac{\left\langle 0\left|\hat{V}^{\omega_{1}}\right| n\right\rangle\left\langle n\left|\hat{V}^{\omega_{2}}\right| 0\right\rangle}{\left(\omega_{n 0}-\omega_{2}\right)^{2}+\gamma_{n 0}^{2}}\right. \\
& \left.-\frac{\left\langle 0\left|\hat{V}^{\omega_{2}}\right| n\right\rangle\langle n| \hat{V}^{\omega_{1}|0\rangle}}{\left(\omega_{n 0}+\omega_{2}\right)^{2}+\gamma_{n 0}^{2}}\right] .
\end{aligned}
$$

The rate of absorbed energy to first order can thereby be written as

$$
\frac{d W}{d t}=-i \mathcal{N} \sum_{\omega_{1}, \omega_{2}} \omega_{1} G\left(\omega_{1} ; \omega_{2}\right) e^{-i\left(\omega_{1}+\omega_{2}\right) t} .
$$

Time averaging then gives

$$
\begin{aligned}
\left\langle\frac{d W}{d t}\right\rangle_{T} & =i \omega \mathcal{N}[G(-\omega ; \omega)-G(\omega ;-\omega)] \\
& =2 \omega \mathcal{N} \operatorname{Im}[-G(-\omega ; \omega)]
\end{aligned}
$$

where we have exploited the symmetry properties of the real and imaginary components of $G(-\omega ; \omega)$,

$$
\begin{gathered}
\operatorname{Re}[G(-\omega ; \omega)]=\operatorname{Re}[G(\omega ;-\omega)] \\
\operatorname{Im}[G(-\omega ; \omega)]=-\operatorname{Im}[G(\omega ;-\omega)] .
\end{gathered}
$$

This expression corresponds to an experimental setup, where the incident radiation has a well-defined polarization and the molecules have a fixed orientation with respect to the radiation field. By comparison with the first term in Eq. (17), the linear absorption cross section, defined as $\sigma(\omega)=\alpha(\omega) / \mathcal{N},{ }^{36}$ becomes equal to

$$
\sigma(\omega)=\frac{4 \omega}{\varepsilon_{0} c \mathcal{E}_{0}^{2}} \operatorname{Im}[-G(-\omega ; \omega)] .
$$

We stress that the response function involving the full fieldmatter interaction operator and also residues of this response function are inherently gauge-origin invariant (see Appendix A), and our formulation thus leads to a physical observable that is independent of the gauge origin. Furthermore, we note that $G(-\omega ; \omega)$ collects the contributions to absorption and dispersion from all higher-order combinations of multipole operators.
Finally, by applying the identity

$$
\lim _{\gamma \rightarrow 0}\left\{\operatorname{Im}\left[\frac{A}{B-i \gamma}\right]\right\}=A \pi \delta(B)
$$

to the linear cross section in Eq. (29), we recover the resonance-divergent result ${ }^{48,49}$

$$
\lim _{\gamma \rightarrow 0} \sigma(\omega)=\frac{4 \pi^{2} \alpha}{e^{2} \omega} \sum_{n>0}\left[\delta\left(\omega-\omega_{n 0}\right)\left|\mathrm{TM}_{\mathrm{BED}}^{0 \rightarrow n}\right|^{2}\right],
$$

where $\alpha=e^{2} /\left(4 \pi \varepsilon_{0} \hbar c\right)$ is the dimensionless fine structure constant and $\mathrm{TM}_{\mathrm{BED}}^{0 \rightarrow n}$ is the transition moment associated with the excitation $0 \rightarrow n$,

$$
\mathrm{TM}_{\mathrm{BED}}^{0 \rightarrow n}=\frac{e}{m_{e}}\left\langle 0\left|\sum_{i=1}^{N} e^{-i \boldsymbol{k} \cdot \boldsymbol{r}_{i}} \boldsymbol{\epsilon} \cdot \boldsymbol{p}_{i}\right| n\right\rangle .
$$

In the following, we label results based on the full field-matter interaction operator by "BED." These results are to be understood as exact, not with regard to the underlying electronic structure method but with regard to the semi-classical description of the light-matter interaction.

At low photon energies, BED corrections to electricdipole-allowed transitions are anticipated to reduce the transition moment norm, i.e., $\left|\mathrm{TM}_{\mathrm{BED}}^{0 \rightarrow n}\right|<\left|\mathrm{TM}_{\mathrm{ED}}^{0 \rightarrow n}\right|$. At high energies and in spatially extended systems, where multiple wavelengths occur within the dimension of the transition density, the situation becomes more complicated and this may no longer hold, as in the case of the trans-polyenes in Sec. V D.

\section{Equation of motion for the complex linear response function}

We derive the equation of motion of the damped linear response function in the frequency domain from the sum-overstates expression,

$$
\begin{aligned}
\left\langle\left\langle\hat{V}^{-\omega} ; \hat{V}^{\omega}\right\rangle\right\rangle= & -\frac{1}{\hbar} \sum_{n>0}\left[\frac{\left\langle 0\left|\hat{V}^{-\omega}\right| n\right\rangle\left\langle n\left|\hat{V}^{\omega}\right| 0\right\rangle}{\omega_{n 0}-\omega-i \gamma_{n 0}}\right. \\
& \left.+\frac{\left\langle 0\left|\hat{V}^{\omega}\right| n\right\rangle\left\langle n\left|\hat{V}^{-\omega}\right| 0\right\rangle}{\omega_{n 0}+\omega+i \gamma_{n 0}}\right] .
\end{aligned}
$$

Making use of the fact that $\frac{a b}{c+d}=\frac{a b}{c}-\frac{d}{c} \frac{a b}{c+d}$, the first term can be rewritten as

$$
\begin{aligned}
& \frac{\left\langle 0\left|\hat{V}^{-\omega}\right| n\right\rangle\left\langle n\left|\hat{V}^{\omega}\right| 0\right\rangle}{\omega_{n 0}-\omega-i \gamma_{n 0}} \\
& =\frac{1}{\omega}\left[-\left\langle 0\left|\hat{V}^{-\omega}\right| n\right\rangle\left\langle n\left|\hat{V}^{\omega}\right| 0\right\rangle+\omega_{n 0} \frac{\left\langle 0\left|\hat{V}^{-\omega}\right| n\right\rangle\left\langle n\left|\hat{V}^{\omega}\right| 0\right\rangle}{\omega_{n 0}-\omega-i \gamma_{n 0}}\right. \\
& \left.\quad-i \gamma_{n 0} \frac{\left\langle 0\left|\hat{V}^{-\omega}\right| n\right\rangle\left\langle n\left|\hat{V}^{\omega}\right| 0\right\rangle}{\omega_{n 0}-\omega-i \gamma_{n 0}}\right] .
\end{aligned}
$$

Applying the same rule to the second term as well as the resolution of the identity, the complex linear response function may be written as

$$
\begin{aligned}
\left\langle\left\langle\hat{V}^{-\omega} ; \hat{V}^{\omega}\right\rangle\right\rangle= & \frac{1}{\hbar \omega}\left[\left\langle 0\left|\left[\hat{V}^{-\omega}, \hat{V}^{\omega}\right]\right| 0\right\rangle+\left\langle\left\langle\left[\hat{V}^{-\omega}, \hat{H}_{0}\right] ; \hat{V}^{\omega}\right\rangle\right\rangle\right. \\
& +i \sum_{n>0} \gamma_{n 0}\left\{\frac{\left\langle 0\left|\hat{V}^{-\omega}\right| n\right\rangle\left\langle n\left|\hat{V}^{\omega}\right| 0\right\rangle}{\omega_{n 0}-\omega-i \gamma_{n 0}}\right. \\
& \left.\left.+\frac{\left\langle 0\left|\hat{V}^{\omega}\right| n\right\rangle\left\langle n\left|\hat{V}^{-\omega}\right| 0\right\rangle}{\omega_{n 0}+\omega+i \gamma_{n 0}}\right\}\right]
\end{aligned}
$$


where $\hat{H}_{0}$ is the electronic Hamiltonian in the absence of the electromagnetic radiation. Compared to the equation of motion for the linear response function in the undamped case, ${ }^{43}$ this expression contains a third sum-over-states term in which the contribution from each state to the response function is weighted by the corresponding damping parameter.

It is a common practice ${ }^{5}$ to choose a common damping term for all excited states, i.e., $\gamma_{n 0}=\gamma$. Upon introducing this approximation, the equation of motion for the damped linear response function reduces to

$$
\begin{aligned}
\hbar(\omega+i \gamma) & \left\langle\left\langle\hat{V}^{-\omega} ; \hat{V}^{\omega}\right\rangle\right\rangle \\
& =\left\langle 0\left|\left[\hat{V}^{-\omega}, \hat{V}^{\omega}\right]\right| 0\right\rangle+\left\langle\left\langle\left[\hat{V}^{-\omega}, \hat{H}_{0}\right] ; \hat{V}^{\omega}\right\rangle\right\rangle,
\end{aligned}
$$

which takes a form similar to the undamped counterpart.

As a special case, we consider the electric dipole approximation $\left(e^{i k \cdot r} \approx 1\right)$, where the equality can be used to relate the linear response function in the velocity representation to the well-known dipole length representation. To this end, we apply the exact state commutation relation between the position and momentum operators $\left[\hat{\boldsymbol{r}}, \hat{H}_{0}\right]=i \hbar \hat{\boldsymbol{p}} / m_{e}$, the symmetry properties of the response function, and invoke Eq. (36) twice. Finally, we obtain

$$
\left\langle\left\langle\hat{p}_{\alpha} ; \hat{p}_{\beta}\right\rangle\right\rangle_{\omega}=m_{e}\left[m_{e}(\omega+i \gamma)^{2}\left\langle\left\langle\hat{r}_{\alpha} ; \hat{r}_{\beta}\right\rangle_{\omega}-N \delta_{\alpha \beta}\right],\right.
$$

which, separated into real and imaginary components, gives

$$
\begin{aligned}
\operatorname{Re}\left\langle\left\langle\hat{p}_{\alpha} ; \hat{p}_{\beta}\right\rangle\right\rangle_{\omega}= & -m_{e}\left[N \delta_{\alpha \beta}-m_{e}\left(\omega^{2}-\gamma^{2}\right) \operatorname{Re}\left\langle\left\langle\hat{r}_{\alpha} ; \hat{r}_{\beta}\right\rangle\right\rangle_{\omega}\right. \\
& \left.+2 m_{e} \omega \gamma \operatorname{Im}\left\langle\left\langle\hat{r}_{\alpha} ; \hat{r}_{\beta}\right\rangle\right\rangle_{\omega}\right] \\
\operatorname{Im}\left\langle\left\langle\hat{p}_{\alpha} ; \hat{p}_{\beta}\right\rangle\right\rangle_{\omega}= & m_{e}^{2}\left[2 \omega \gamma \operatorname { R e } \left\langle\left\langle\hat{r}_{\alpha} ; \hat{r}_{\beta}\right\rangle_{\omega}\right.\right. \\
& \left.+\left(\omega^{2}-\gamma^{2}\right) \operatorname{Im}\left\langle\left\langle\hat{r}_{\alpha} ; \hat{r}_{\beta}\right\rangle\right\rangle_{\omega}\right] .
\end{aligned}
$$

We investigate this sum rule numerically in Sec. V.

\section{IMPLEMENTATION}

The complex linear response function in Eq. (23) has been implemented at the TD-DFT level of theory using the firstorder CPP formalism, ${ }^{37,38}$ in which the explicit reference to the excited states in the sum-over-states expression is replaced by a matrix equation. In contrast to traditional resonant-divergent response theory, where absorption properties are evaluated by means of the eigenvectors of the electronic Hessian matrix found by a bottom-up approach, the CPP approach allows us to study core excitations without restrictions on the excitation manifold. Below, we outline the implementation of the field-matter interaction operator and its coupling to the CPP approach in the DALTON program package, ${ }^{50,51}$ using the most recent CPP algorithm. ${ }^{52,53}$ The reader is referred to Refs. 37 and 38 for a detailed description of the CPP approach.

In the case of TD-DFT, the damped linear response function, involving the field-matter interaction operator, can be written as

$$
\begin{aligned}
\left.\left\langle\hat{V}^{-\omega} ; \hat{V}^{\omega}\right\rangle\right\rangle & =-\left(\boldsymbol{\eta}_{R}^{\omega}+i \boldsymbol{\eta}_{I}^{\omega}\right)^{\dagger}\left(\boldsymbol{P}_{R}^{\omega}+i \boldsymbol{P}_{I}^{\omega}\right) \\
& =-\left(\boldsymbol{\eta}_{R}^{\omega \dagger} \boldsymbol{P}_{R}^{\omega}+\boldsymbol{\eta}_{I}^{\omega \dagger} \boldsymbol{P}_{I}^{\omega}\right)-i\left(\boldsymbol{\eta}_{R}^{\omega \dagger} \boldsymbol{P}_{I}^{\omega}-\boldsymbol{\eta}_{I}^{\omega \dagger} \boldsymbol{P}_{R}^{\omega}\right) .
\end{aligned}
$$

Here, $\boldsymbol{P}^{\omega}$ is the first-order response vector and $\boldsymbol{\eta}^{\omega}$ is the property gradient defined by

$$
\boldsymbol{\eta}^{\omega}=\frac{2 i \omega}{\mathcal{E}_{0}}\left\langle 0\left|\left[\hat{\mathbf{q}}, \hat{V}^{\omega}\right]\right| 0\right\rangle,
$$

where $\hat{\mathbf{q}}$ is the vector containing the usual de-excitation and excitation operators. ${ }^{43}$ Subscripts $R$ and $I$ refer to the real and imaginary components, respectively, of the given vector. Going beyond the ED approximation thus introduces a complexvalued property gradient that depends on the frequency of the incident radiation field. It is therefore necessary to re-evaluate the property gradient for each input frequency/response vector. The complex response vector is obtained by solving the firstorder damped linear response equations,

$$
\left(\boldsymbol{E}^{[2]}-\hbar(\omega+i \gamma) \boldsymbol{S}^{[2]}\right)\left(\boldsymbol{P}_{R}^{\omega}+i \boldsymbol{P}_{I}^{\omega}\right)=\boldsymbol{\eta}_{R}^{\omega}+i \boldsymbol{\eta}_{I}^{\omega},
$$

where $\boldsymbol{E}^{[2]}$ is the electronic Hessian, and $\boldsymbol{S}^{[2]}$ is a metric matrix. The explicit expressions for these matrices may be found in Eqs. (50) and (51) of Ref. 54. The construction of the property gradients $\boldsymbol{\eta}^{ \pm \omega}$ of the field-matter interaction operator requires the evaluation of integrals of the form

$$
\left\langle\chi_{\mu}\left|\hat{v}^{ \pm \omega}\right| \chi_{\nu}\right\rangle= \pm \frac{e \mathcal{E}_{0}}{2 i \omega m_{e}}\left\langle\chi_{\mu}\left|e^{ \pm i \boldsymbol{k} \cdot \boldsymbol{r}}(\boldsymbol{\epsilon} \cdot \hat{\boldsymbol{p}})\right| \chi_{\nu}\right\rangle,
$$

where $\chi_{\mu}$ and $\chi_{v}$ denote the atomic orbitals (AOs) and $\hat{v}^{ \pm \omega}$ the one-electron interaction operator [see Eq. (8)]. The complex exponential is separated according to Euler's formula into a real component

$$
\begin{aligned}
\operatorname{Re}\left[e^{ \pm i k \cdot r}\right]= & \cos \left(k_{x} x\right) \cos \left(k_{y} y\right) \cos \left(k_{z} z\right) \\
& -\cos \left(k_{x} x\right) \sin \left(k_{y} y\right) \sin \left(k_{z} z\right) \\
& -\sin \left(k_{x} x\right) \cos \left(k_{y} y\right) \sin \left(k_{z} z\right) \\
& -\sin \left(k_{x} x\right) \sin \left(k_{y} y\right) \cos \left(k_{z} z\right)
\end{aligned}
$$

and an imaginary component

$$
\begin{aligned}
\operatorname{Im}\left[e^{ \pm i \boldsymbol{k} \cdot \boldsymbol{r}}\right]= & \pm\left(\cos \left(k_{x} x\right) \cos \left(k_{y} y\right) \sin \left(k_{z} z\right)\right. \\
& +\cos \left(k_{x} x\right) \sin \left(k_{y} y\right) \cos \left(k_{z} z\right) \\
& +\sin \left(k_{x} x\right) \cos \left(k_{y} y\right) \cos \left(k_{z} z\right) \\
& \left.-\sin \left(k_{x} x\right) \sin \left(k_{y} y\right) \sin \left(k_{z} z\right)\right) .
\end{aligned}
$$

Finally, multiplication with the linear momentum operator gives the expression for the real and imaginary components of the one-electron field-matter interaction operator,

$$
\begin{aligned}
\hat{v}^{\omega} & =-\frac{e \hbar \mathcal{E}_{0}}{2 \omega m_{e}}\left(\operatorname{Re}\left[e^{i k \cdot r}\right]+i \operatorname{Im}\left[e^{i k \cdot r}\right]\right)(\boldsymbol{\epsilon} \cdot \nabla), \\
\hat{v}^{-\omega} & =\frac{e \hbar \mathcal{E}_{0}}{2 \omega m_{e}}\left(\operatorname{Re}\left[e^{i k \cdot r}\right]-i \operatorname{Im}\left[e^{i k \cdot r}\right]\right)(\boldsymbol{\epsilon} \cdot \nabla) .
\end{aligned}
$$

Due to the (anti)symmetric properties of cosine (sine) functions, the property gradient for the negative frequency can be straightforwardly obtained from the integrals associated with the positive frequency by simply adjusting the signs. A detailed description of the evaluation of the AO integrals over the field-matter interaction operator in Eq. (46) can be found in Appendix B.

The linear absorption can alternatively be formulated within the infinite lifetime approximation $(\gamma=0)$ using Fermi's golden rule. ${ }^{40,49}$ Excitation energies and transition 
moments are here identified on the basis of a pole and residue analysis of the resonant-divergent linear response function of the field-matter interaction operator. In short, excitation energies are found by solving the generalized eigenvalue problem

$$
\left(\boldsymbol{E}^{[2]}-\hbar \omega_{n 0} \boldsymbol{S}^{[2]}\right) \boldsymbol{X}_{n}=0
$$

and transition moments obtained by contracting excitation vectors $\left(\boldsymbol{X}_{n}\right)$ with property gradients

$$
\mathrm{TM}^{0 \rightarrow n}=\boldsymbol{X}_{n}^{\dagger}\left(\omega_{n 0}\right) \boldsymbol{\eta}^{ \pm \omega}
$$

More detailed information is found in, e.g., Refs. 43 and 55. We have complemented the damped response theory based implementation of linear absorption based on the field-matter interaction operator with the evaluation of the corresponding transition moment at both the Hartree-Fock, TD-DFT, and multiconfigurational self-consistent field (MCSCF) levels of theories. We note that contrary to the CPP formulation, the excitation energies are required to construct the resonant fieldmatter interaction operator. On the other hand, it is possible to contract a solution vector associated with a specific excitation energy with property gradients of the field-matter interaction operator of different frequency, a functionality that will be exploited in Sec. V D. An important point to note in this context is that we consider only squared transition moments to ensure origin-independent results, see Appendix A.

\section{COMPUTATIONAL DETAILS}

We have computed the NEXAFS spectra at the carbon $K$ edge for a series of trans-polyenes. The molecular geometries were optimized using the Gaussian $09 \operatorname{code}^{56}$ at the DFT level of theory, employing the B3LYP ${ }^{57-60}$ exchange-correlation functional and the cc-pVDZ ${ }^{61}$ basis set. For the property calculations, a proper description of the long-range Coulomb interactions between initial and final electronic states in the absorption process has been ensured by the use of the CAM-B3LYP ${ }_{100 \%}$ functional. This is a modified version of the Coulomb-attenuated method CAM-B3LYP ${ }^{62}$ functional taking into account $100 \%$ Hartree-Fock Coulombic interactions in the long-range limit ( $\alpha=0.19, \beta=0.81$, and $\mu=0.33$ ). As compared to the original parameterization, this choice has been found superior for the description of core excitations. ${ }^{39}$ Absorption cross sections for both the full field-matter interaction operator and within the ED approximation were determined with a lifetime broadening $\gamma=1000 \mathrm{~cm}^{-1}$ (or $0.1240 \mathrm{eV})$. All calculations were performed under the constraint of $\mathrm{C}_{2 h}$ symmetry.

Excitation energies and transition moment norms of the full field-matter interaction operator as well as within the ED approximation for the alkaline earth metals were obtained from a pole and residue analysis of the resonantdivergent linear response function. For the lowest singlet valence excitations in $\mathrm{Mg}$, the calculations were performed at the MCSCF level of theory, considering a complete active space consisting of two electrons in four orbitals CAS $(2,4)$. An uncontracted basis set was used, with the exponents for the core and valence orbitals taken from the q-aug-cc-pVQZ basis set by Woon and Dunning. ${ }^{63}$ Core excitations for $\mathrm{Mg}$,
$\mathrm{Ca}, \mathrm{Sr}, \mathrm{Ba}$, and $\mathrm{Ra}$ were described at the CAM-B3LYP $100 \%$ level using an uncontracted basis set with exponents adapted from the ANO- $\mathrm{RCC}^{64}$ basis set. To reach the core excitations within the resonant-divergent linear response formalism, we used the restricted-channel approximation, ${ }^{39}$ in which only a subblock of the electronic Hessian, involving excitations from the $1 s$-orbital to any of the virtual orbitals, was considered.

Unless stated otherwise, the propagation direction of the radiation field was taken to be along the $z$-axis, i.e., $\boldsymbol{k}=k \boldsymbol{e}_{z}$ and the polarization vector to be along the $x$-direction $\left(\boldsymbol{\epsilon}=\boldsymbol{e}_{x}\right)$, where $\boldsymbol{e}_{\alpha}$ is a unit vector along the Cartesian $\alpha$-axis. Equations (44) and (45) then simplifies to

$$
\operatorname{Re}\left[e^{ \pm i k \cdot r}\right]=\cos (k z) ; \quad \operatorname{Im}\left[e^{ \pm i k \cdot r}\right]= \pm \sin (k z) .
$$

The property calculations were performed with a locally modified version of the DALTON program, ${ }^{50,51}$ which includes the implementation of the property gradient of the field-matter interaction operator within both the damped and undamped linear response frameworks.

\section{RESULTS AND DISCUSSION}

\section{A. Sum rules and gauge dependence: A numerical study}

We have investigated numerically the equivalency between the linear response functions in the dipole length and dipole velocity gauges, Eqs. (38) and (39), respectively. Results are shown for $\mathrm{He}$ in Table I using basis sets of increasing size. As can be seen from an explicit evaluation of the Thomas-Reiche-Kuhn rule $S(0)=\langle 0|[\hat{\boldsymbol{r}}, \hat{\boldsymbol{p}}]| 0\rangle$, which is used in place of the number of electrons $N$, the commutator relation between the position and linear momentum operator, and thus Eq. (37), is not exact in finite basis set calculations. Accordingly, the correspondence between the values obtained by combining Eqs. (38) and (39) with the dipole-dipole polarizability in length gauge, and the real and imaginary parts of the dipole-dipole polarizability in the velocity gauge progressively improve as one approaches the basis set limit. Furthermore, by shifting the origin along the individual coordinate axes, we confirmed numerically the origin-independence of the response function of the full field-matter interaction operator (data not shown).

\section{B. Valence excitations: Magnesium}

To illustrate the calculation of the transition moment norms of the field-matter interaction operator and to verify the implementation of the property gradient, we begin by considering the lowest singlet valence excitations in $\mathrm{Mg}$. We perform a residue analysis of the undamped linear response function of the field-matter interaction operator, where $\hat{V}^{\omega_{n} 0}$ is evaluated at an angular frequency $\omega_{n 0}=\Delta E_{n 0} \hbar^{-1}$ matching the excitation energy of the $n$th transition. In this lowenergy region $\left(\Delta E_{n 0}<10 \mathrm{eV}\right)$, the spatial variations in the field-matter interaction operator across the extent of the transitions are limited, and the effect of going beyond the ED approximation is therefore expected to be significant only for electric-dipole-forbidden transitions. For the present choice of 
TABLE I. Hartree-Fock calculations on helium probing the equation-of-motion equivalency in Eq. (37) for a diagonal element of the electronic dipole-dipole polarizability. The response functions are evaluated at $\omega=1.0$ a.u. and $\gamma=1000 \mathrm{~cm}^{-1}$. All quantities are provided in a.u.

\begin{tabular}{|c|c|c|c|c|c|c|c|}
\hline \multirow[b]{2}{*}{ Basis set } & \multirow[b]{2}{*}{$S(0)$} & \multicolumn{2}{|c|}{$-\left\langle\left\langle\hat{r}_{\alpha} ; \hat{r}_{\alpha}\right\rangle\right\rangle_{\omega}$} & \multirow[b]{2}{*}{ Equation (38) } & \multirow[b]{2}{*}{ Equation (39) } & \multicolumn{2}{|c|}{$-\left\langle\left\langle\hat{p}_{\alpha} ; \hat{p}_{\alpha}\right\rangle\right\rangle_{\omega}$} \\
\hline & & $\operatorname{Re}$ & $\operatorname{Im}$ & & & $\operatorname{Re}$ & $\mathrm{Im}$ \\
\hline aug-cc-pVDZ & 1.971 & 19.726 & 2.710 & 21.672 & 2.890 & 21.320 & 2.844 \\
\hline d-aug-cc-pVDZ & 1.971 & 2.573 & 0.119 & 4.544 & 0.142 & 4.439 & 0.140 \\
\hline t-aug-cc-pVTZ & 1.993 & 1.552 & 0.130 & 3.543 & 0.144 & 3.491 & 0.143 \\
\hline q-aug-cc-pVQZ & 1.999 & 2.266 & 0.219 & 4.263 & 0.239 & 4.215 & 0.239 \\
\hline q-aug-cc-pV5Z & 1.999 & 4.277 & 0.516 & 6.272 & 0.555 & 6.245 & 0.554 \\
\hline q-aug-cc-pV6Z & 2.000 & 4.101 & 0.550 & 6.096 & 0.588 & 6.095 & 0.588 \\
\hline
\end{tabular}

propagation direction, the cosine and sine terms of the operator cannot mix, which means that the electric-dipole and all higher odd-order contributions arise from the first term of Eq. (44), whereas all even-order contributions are contained in the first term of Eq. (45).

In Table II, we report the norm of the transition moments for the valence excitations of $\mathrm{Mg}$ as obtained both within and beyond the ED approximation. We also include the corresponding experimental transition energies. Only transitions within a degenerate set that possess a nonzero transition moment are listed. As seen from a comparison of the ED and BED transition moments, the electric-dipole-allowed $3 s \rightarrow p$ transitions are dominated by the zeroth-order term in the cosine expansion. The higher-order contributions included in the field-matter interaction operator are, for the $s \rightarrow p$ transitions, orders of magnitudes smaller (in the range of $10^{-6}-10^{-8}$ ) than the electric-dipole contribution. The relative importance of the zeroth- and higher-order terms agrees well with what can be expected for the leading correction $\left(\frac{1}{2} k^{2}\left\langle\hat{r}_{n p}\right\rangle^{2}\right.$, where we obtain $\left\langle\hat{r}_{3 p}\right\rangle=3.40 a_{0}$ from an atomic HF calculation of the $3 s 3 p$ excited state) from the electric-dipole and electricoctupole/magnetic-quadrupole cross terms. In principle, the electric and magnetic contributions can be distinguished by treating the symmetric and antisymmetric parts of the integrals separately. However, such a separation introduces an origindependence in the individual terms, ${ }^{10}$ something that we will avoid in the present work.

TABLE II. Excitation energies $\Delta E_{n 0}(\mathrm{eV})$ and norm of transition moments (a.u.) within and beyond the ED approximation, $\left|\mathrm{TM}_{\mathrm{ED}}^{0 \rightarrow n}\right|$ and $\left|\mathrm{TM}_{\mathrm{BED}}^{0 \rightarrow n}\right|$, respectively, together with the difference $\Delta\left|\mathrm{TM}^{0 \rightarrow n}\right|$ for the lowest singlet transitions in $\mathrm{Mg}$, computed at the CAS(2,4)/q-aug-cc-pVQZ level of theory.

\begin{tabular}{lccccc}
\hline \hline Transition & $\Delta E_{n 0}$ & Expt. $^{\mathrm{a}}$ & $\left|\mathrm{TM}_{\mathrm{BED}}^{0 \rightarrow n}\right|$ & $\left|\mathrm{TM}_{\mathrm{ED}}^{0 \rightarrow n}\right|$ & $\Delta\left|\mathrm{TM}^{0 \rightarrow n}\right|$ \\
\hline $3 s \rightarrow 3 p_{x}$ & 4.317 & 4.346 & $3.74 \times 10^{-1}$ & $3.74 \times 10^{-1}$ & $-1.79 \times 10^{-6}$ \\
$3 s \rightarrow 4 s$ & 5.435 & 5.394 & $\ldots$ & $\ldots$ & $\ldots$ \\
$3 s \rightarrow 3 d_{x z}$ & 5.644 & 5.753 & $7.60 \times 10^{-4}$ & $\ldots$ & $7.60 \times 10^{-4}$ \\
$3 s \rightarrow 4 p_{x}$ & 6.130 & 6.118 & $1.02 \times 10^{-1}$ & $1.02 \times 10^{-1}$ & $1.29 \times 10^{-8}$ \\
$3 s \rightarrow 5 s$ & 6.550 & 6.516 & $\ldots$ & $\ldots$ & $\ldots$ \\
$3 s \rightarrow 4 d_{x z}$ & 6.577 & 6.588 & $1.61 \times 10^{-4}$ & $\ldots$ & $1.61 \times 10^{-4}$ \\
$3 s \rightarrow 4 f_{x z^{2}}$ & 6.811 & 6.779 & $4.16 \times 10^{-7}$ & $\ldots$ & $4.16 \times 10^{-7}$ \\
$3 s \rightarrow 4 f_{x\left(x^{2}-3 y^{2}\right)}$ & 6.811 & 6.779 & $4.86 \times 10^{-7}$ & $\ldots$ & $4.86 \times 10^{-7}$ \\
$3 s \rightarrow 5 p_{x}$ & 6.823 & 6.783 & $4.30 \times 10^{-2}$ & $4.30 \times 10^{-2}$ & $9.56 \times 10^{-8}$ \\
$3 s \rightarrow 6 s$ & 7.006 & 6.966 & $\ldots$ & $\ldots$ & $\ldots$ \\
$3 s \rightarrow 5 d_{x z}$ & 7.009 & 6.981 & $2.10 \times 10^{-5}$ & $\ldots$ & $2.10 \times 10^{-5}$ \\
\hline \hline
\end{tabular}

a Taken from Ref. 65.
For the electric-dipole-forbidden transitions, higher-order contributions are of course required in the calculation to obtain a nonzero transition probability. The dominant contribution to the $3 s \rightarrow d$ transitions comes from the leading term in the sine expansion, i.e., the electric-quadrupole/magnetic-dipole contribution. Accordingly, the $3 s \rightarrow d$ transition moments differ from the electric-dipole-allowed counterparts by a factor of approximately $10^{-4}$. The $3 s \rightarrow 4 f$ transitions are even weaker, i.e., of the same order of magnitude as the electricoctupole/magnetic-quadrupole correction to the $3 s \rightarrow p$ transitions. Note that the slightly different transition moments for the two degenerate $3 s \rightarrow 4 f$ transitions result from the specific choice of propagation and polarization vectors combined with the fact that no rotational averaging has been performed. Finally, we note that the totally symmetric $s \rightarrow s$ transitions are entirely forbidden due to the absence of a totally symmetric component in the field-matter interaction operator, which is a consequence of the orthogonality between the polarization and propagation vectors. Such transitions do become allowed, though, when extending from electromagnetic to electroweak interactions. ${ }^{66}$

\section{C. $K$-edge NEXAFS of alkaline earth metals}

In this section, we assess the spectral range (soft to hard X-ray region) in which the ED approximation can be safely employed to describe the interaction between atoms and electromagnetic radiation. The validity of the ED approximation depends on the relative dimensions of field wavelengths and atoms, or, more specifically, on the particular electronic transitions that are being probed. BED corrections become increasingly important when $k r$ increases, and when exceeding unity, the ED approximation will break down. While transition energies of core excitations increase as one goes down a group in the periodic table, the spatial confinement of the excitation becomes progressively stronger. The $k r$ values are thus dictated by the relative behavior of two competing factors with respect to the atomic number, and, in order to probe the validity of the ED approximation, we therefore examine the range of $k r$ values relevant within the periodic table. We consider the lowest dipole-allowed $1 s \rightarrow n p_{x}$ transitions and the corresponding energetically close-lying $1 s \rightarrow m d_{x z}$ transitions (dipole-forbidden but quadrupole-allowed) in the alkaline earth metals $(\mathrm{Mg}, \mathrm{Ca}, \mathrm{Sr}, \mathrm{Ba}$, and $\mathrm{Ra})$, where $m=n-1$ for all but $\mathrm{Mg}$, for which $m=n$. We again stress 
that relativistic effects are not taken into account, but will be pursued in future work. Note also that the atomic symmetry restricts any mixing of the odd- and even-order terms in the expansion of the complex exponential in the field-matter interaction operator. In other words, the lowest-order BED correction to electric-dipole-allowed transitions will be quadratic in $k$, corresponding to the contributions from the electricoctupole/magnetic-dipole terms, as discussed in Sec. V B.

To determine the relationship between $k r$ and atomic number $(Z)$, we evaluated numerically the magnitude of the BED corrections to the lowest $1 s \rightarrow n p_{x}$ and $1 s \rightarrow m d_{x z}$ transitions down the $\mathrm{Mg}-\mathrm{Ra}$ group. Figure 1(a) displays the norm of the BED transition moments across the series and compares to the counterparts obtained within the ED approximation. Apart from being essential for the description of electric-dipole-forbidden transitions, we find that the BED transition moments for the quadrupole-allowed $1 s \rightarrow m d_{x z}$ transitions to be larger than the BED corrections for the electric-dipole-allowed transitions, which remain modest across the entire series. For Ra, the transition moment of the $1 s \rightarrow 6 d_{x z}$ transition becomes equal to about 0.15 a.u., which is about one third the size of the corresponding $1 s \rightarrow 7 p_{x}$ transition moment. Assuming the $k r$ values to be similar for the two transitions and recalling that the leading term in the BED corrections to electric-dipole-allowed transitions in atoms is proportional to $k^{2}$ for small values of $k$ while linear in

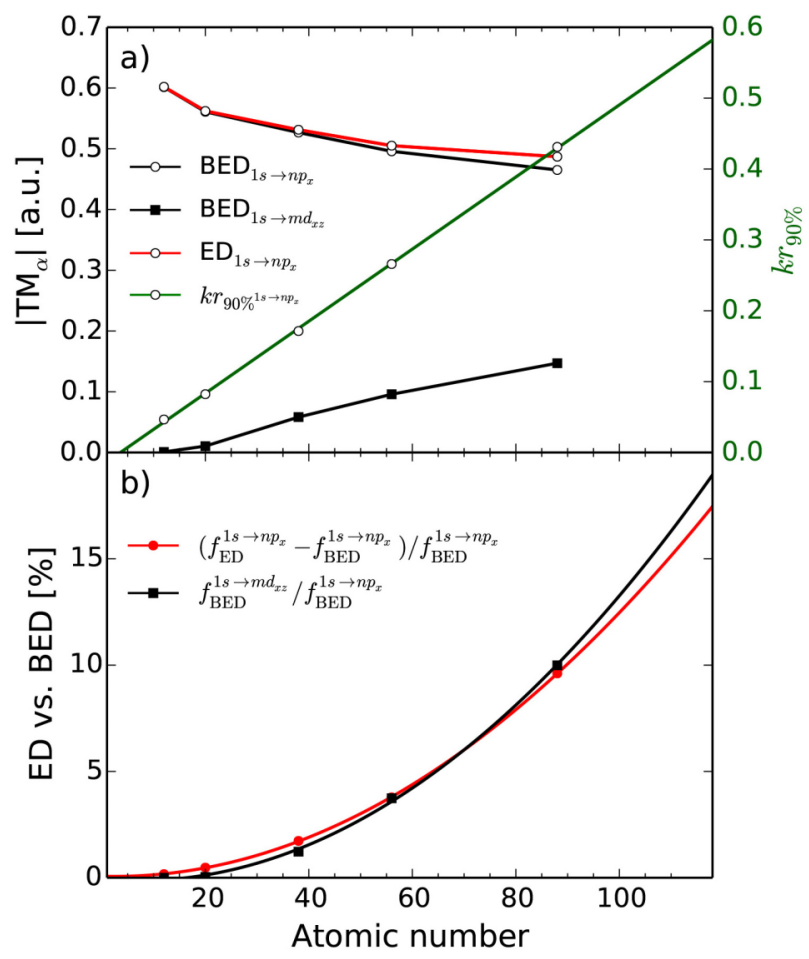

FIG. 1. (a) Norm of the transition moments $\left(\left|\mathrm{TM}_{\alpha}\right|, \alpha=\mathrm{ED}, \mathrm{BED}\right)$ for the $1 s \rightarrow n p_{x}$ and $1 s \rightarrow m d_{x z}$ transitions, respectively, computed within and beyond the ED approximation (left axis) as well as $k r_{90 \%}$ values (right axis; see text for definition) for the $1 s \rightarrow n p_{x}$ transition across group 2, together with the $(Z, k r)$ linear fit $\left(5.1 \times 10^{-3}\right.$ a.u. $\left.{ }^{-1} Z-1.82 \times 10^{-2}\right)$. (b) Estimated error in the oscillator strength of the $1 s \rightarrow n p_{x}$ and $1 s \rightarrow m d_{x z}$ transitions across the series introduced by invoking the ED approximation. The magnitude of the BED corrections to the $1 s \rightarrow m d_{x z}$ transitions is relative to the $1 s \rightarrow n p_{x}$ transition. Lines are quadratic fits of the data. $k$ for quadrupole-allowed transitions, such a behavior suggests that $k r$ is well below unity for the transitions considered in the alkaline earth metals.

Further insight into the modest effect of going beyond the ED approximation can be gained by considering the quantities underlying the transition moments. In this analysis, we restrict our attention to the $1 s \rightarrow n p_{x}$ transitions across the $\mathrm{Mg}-\mathrm{Ra}$ series. Figure 2 depicts the $\phi_{1 s}$ and $\phi_{n p_{x}}$ orbitals along the $x$-axis (polarization direction), where the $\phi_{n p_{x}}$ final states have been constructed as linear combinations of the dominating (weight $>10 \%$ ) orbital contributions to the relevant eigenvectors of the electronic Hessian.

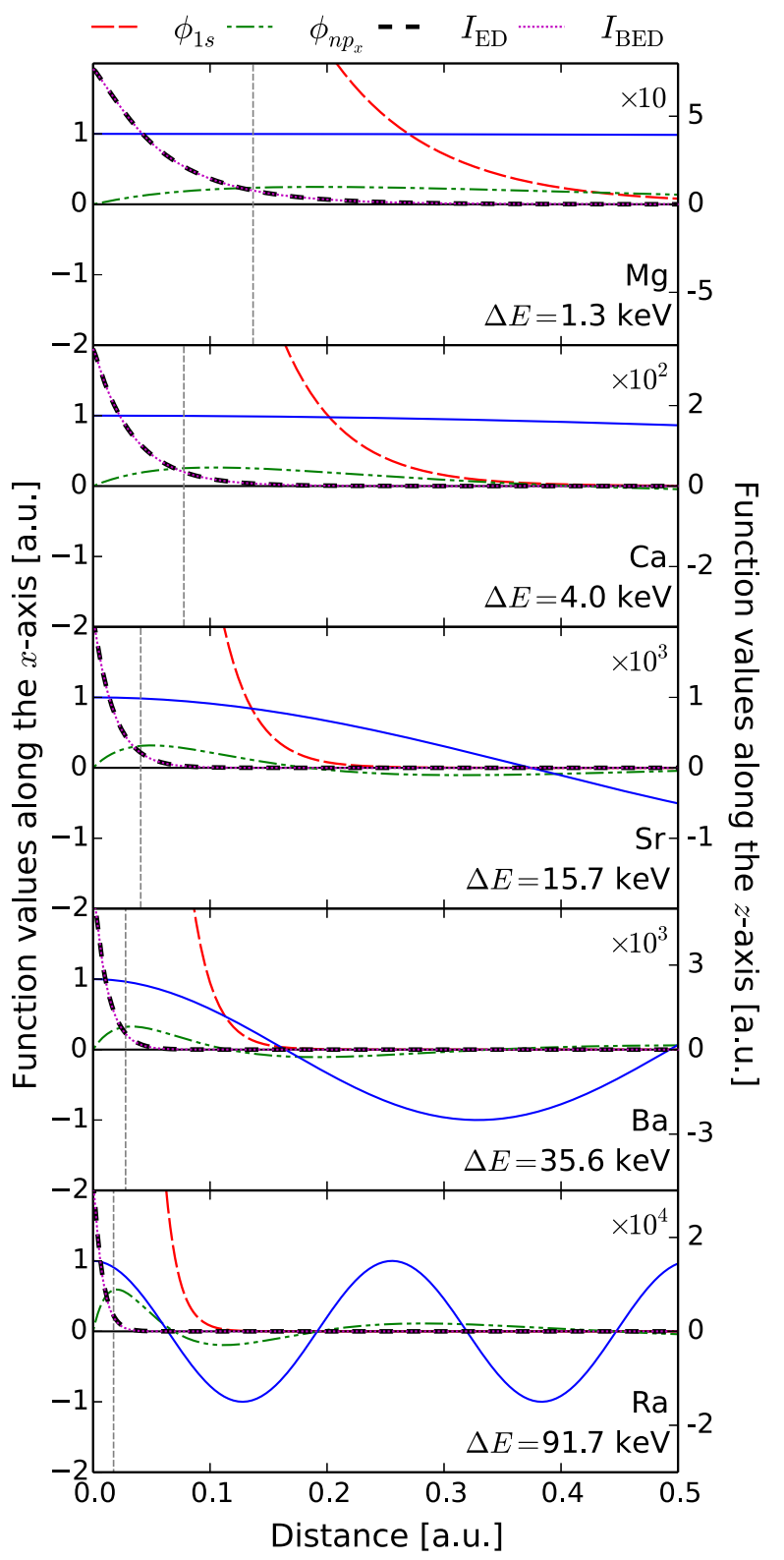

FIG. 2. (Left axis) $1 s$ - and $n p_{x}$-orbitals relevant for the $1 s \rightarrow n p_{x}$ core transitions across the $\mathrm{Mg}-\mathrm{Ra}$ series plotted along the $x$-axis (polarization direction). The excitation fields are illustrated by cosine functions (blue). (Right axis) Integrands of the transition moment integrals within $\left(I_{\mathrm{ED}}\right)$ and beyond $\left(I_{\mathrm{BED}}\right)$ the ED approximation as plotted along the $z$-axis (propagation direction). The excitation fields are likewise plotted along $z$. Vertical lines indicate $r_{90 \%}$ (see text for definition). The excitation energies $(\Delta E)$ are reported next to the distributions. 


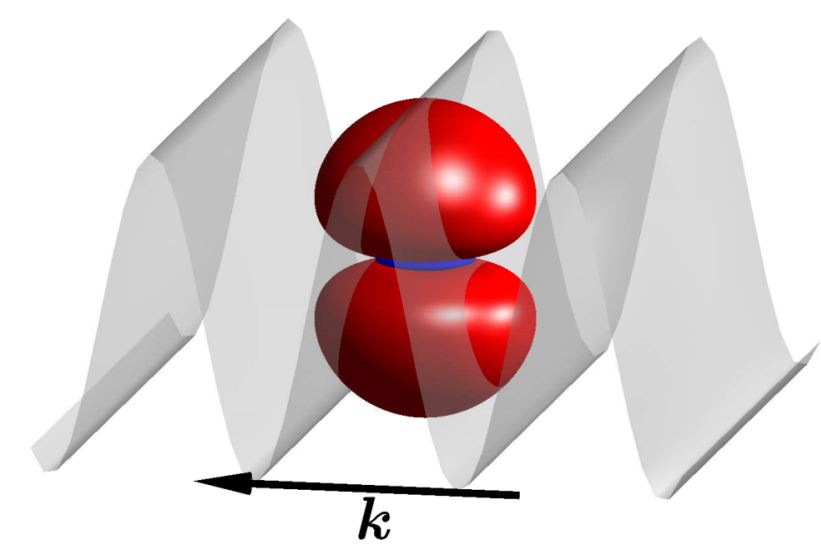

FIG. 3. The integrand $I_{\mathrm{ED}}$ for $\mathrm{Mg}$ (isovalues: \pm 0.8 a.u.) in the presence of a non-resonant monochromatic, linearly polarized field propagating along the $z$-axis $\left(k_{z}=5\right.$ a.u.) with the polarization vector along the $x$-axis.

In addition, we plot the associated integrands $I$ of the transition moment integrals along the $z$-axis (propagation direction) both within and beyond the ED approximation, i.e., $I_{\mathrm{ED}}\left(0,0, z ; k_{z}\right)=\phi_{1 s}(0,0, z) \nabla_{x} \phi_{n p_{x}}(0,0, z)$ (corresponding to a ket-differentiated transition density) and $I_{\mathrm{BED}}\left(0,0, z ; k_{z}\right)=\cos \left(k_{z} z\right) I_{\mathrm{ED}}\left(0,0, z ; k_{z}\right)$, respectively. Note that different scales have been used to plot the orbital distributions (left $y$-axis) and integrands (right $y$-axis). We have also included the corresponding cosine functions to represent the incident excitation field. A three-dimensional representation of the field-matter interaction is given in Figure 3, which illustrates $I_{\mathrm{ED}}$ for $\mathrm{Mg}$ (isovalues: \pm 0.8 a.u.), experiencing an incident radiation field. The wavelength is here shorter compared to the actual excitation wavelength as to clarify the polarization and propagation directions of the field.

The orbital contraction that follows as group 2 is descended is clearly seen in Figure 2. For the $K$-edge transitions, the spatial extension of integrands is effectively limited by the compactness of the exponentially decaying $1 s$-orbital. To get an estimate for the spatial extension of $I_{\mathrm{ED}}$, we have proceeded as follows: A decomposition of the transition moment integrals across the series into contributions introduced by the action of the linear momentum operator on the $n p_{x}$ orbitals showed that the resulting $s$ contribution dominates over the $d_{x^{2}}$-type contribution (zero along the $z$ direction), which is furthermore of opposite sign. The BED corrections to the latter turn out to be of about half the (absolute) size of those arising from the $s$ contribution, and the $\mathrm{BED}$ correction to the total transition moment integral is thus about half of that of the $s$ contribution. A sensible estimate for an upper boundary of the BED corrections can hence be based on the $s$ contribution alone. As estimate for the effective radius of the transition, we therefore use $r_{90 \%}$, corresponding to the radius that encloses $90 \%$ of the $s$ contribution to the integrand along the $z$-direction. These estimates are indicated by vertical bars in Figure 2. As can be seen in the figure, the external field shows only small spatial variations over the extension of $I_{\mathrm{ED}}$, indicated by the $r_{90 \%}$ estimates.

The behavior of the $r_{90 \%}$ estimates with respect to atomic number is displayed in Figure 4. Included is also the trend in the maximum of the $1 s$ radial distributions, $r_{1 s}$, as

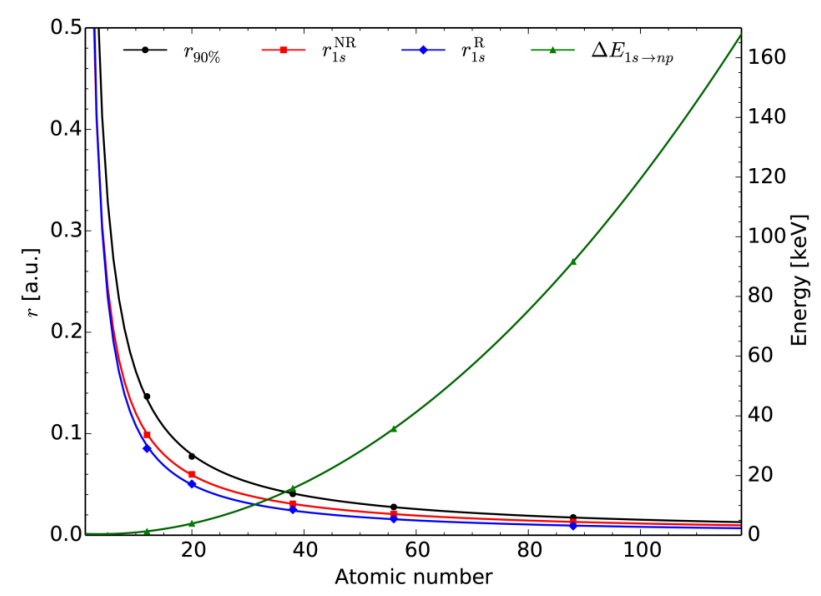

FIG. 4. Trends in $r_{90 \%}$ (left axis; see text for definition) and the excitation energies $\Delta E_{1 s \rightarrow n p}$ (right axis) across the $\mathrm{Mg}, \mathrm{Ca}, \mathrm{Sr}, \mathrm{Ba}$, and $\mathrm{Ra}$ series. The maxima of the $1 s$ radial distribution functions as obtained from nonrelativistic (NR) calculations as well as relativistic (R) Hartree-Fock/Dyall $\mathrm{TZ}^{69}$ calculations based on the Dirac-Coulomb Hamiltonian. ${ }^{70}$ The radii are almost inversely proportional to the atomic number, whereas the excitation energy behaves quadratically. Lines are the corresponding fits.

obtained from non-relativistic calculations across the series. For comparison, we have also included the result of relativistic calculations. As evident, the $r_{90 \%}$ value is almost inversely proportional to the atomic number, as can be expected from the analogy to the radial extension of the orbitals of hydrogenic atoms. In Figure 4, we also provide the excitation energies of the $1 s \rightarrow n p$ transitions across the $\mathrm{Mg}-\mathrm{Ra}$ series to facilitate an assessment of the $k r$ values. Consistent with the similarity to the ionization potential of the $1 s$-orbital in a hydrogenic atom, the transition energy, on the other hand, exhibits a quadratic dependence on the atomic number. The change in transition energies down the group is thus stronger than the associated contraction of the differentiated transition density $I_{\mathrm{ED}}$. As a consequence, the $k r_{90 \%}$ estimate, combining the $r_{90 \%}$ estimate with the norm of the wavevector, depends linearly on the atomic number, as shown in Figure 1(a). Most importantly, we find that the $k r_{90 \%}$ values across group 2 are all below unity. In fact, an extrapolation to the maximum atomic number indicates that the electric-dipole term remains the dominating contribution to the absorption intensities of electric-dipoleallowed transitions throughout the periodic table $(k r<0.6$ for $Z=118$ ).

Having considered the range of $k r$ values that covers absorption spectroscopies across the periodic table, it is interesting to examine the errors that can be expected in the oscillator strength upon invoking the ED approximation. Figure 1(b) illustrates the percentage error in the oscillator strength for the $1 s \rightarrow n p_{x}$ transitions across the series introduced by the ED approximation. For the electric-dipoleforbidden $1 s \rightarrow m d_{x z}$ transition, we report the oscillator strength relative to that for the electric-dipole-allowed transition. In both cases, the error measures behave close to quadratically with respect to $k r$, as anticipated for $k r<1$, and thus likewise with respect to the atomic number. The error in the oscillator strength for the $1 s \rightarrow n p_{x}$ transition due to the ED approximation is about $10 \%$ for $\mathrm{Ra}$ and amounts to at most $20 \%$ within the periodic table. In the more general case, 


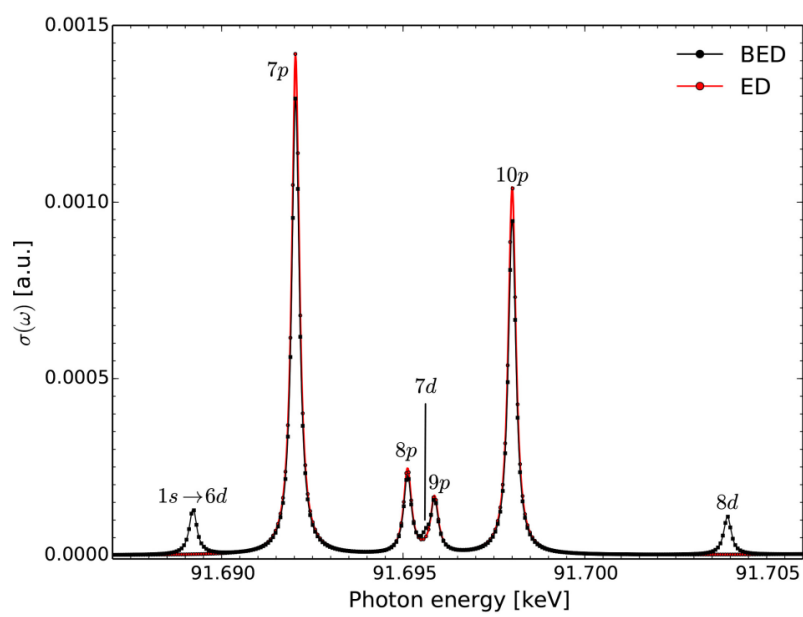

FIG. 5. XAS spectrum of Ra computed within and beyond the ED approximation at the CAM-B3LYP $100 \% / A N O-R C C$ level of theory using a common lifetime broadening parameter $\gamma=1000 \mathrm{~cm}^{-1}$. The experimental $1 s$ ionization energy is $103.922 \mathrm{keV} .^{71}$

where $p-d$ mixing is allowed, an estimate for the additional BED correction stemming from the $d$-admixture (via the sine term) may be obtained from the $f_{\mathrm{BED}}^{1 s \rightarrow m d_{x z}} / f_{\mathrm{BED}}^{1 s \rightarrow n p_{x}}$ ratio and the relative $p-d$ character of the final state.

Before moving to larger molecular systems, we demonstrate the calculation of the $K$-edge NEXAFS spectrum of $\mathrm{Ra}$ using the CPP approach in combination with the field-matter interaction operator implemented in this work. The absorption spectrum for Ra, computed using Eq. (29), is depicted in Figure 5, together with the one obtained within the ED approximation. The absorption peaks arising from electric-dipole-allowed $1 s \rightarrow p_{x}$ transitions can be identified immediately as the overlapping peaks in the ED and BED spectra. This peak assignment is further confirmed by an analysis of the response vectors. The intensities at the peak maxima for these transitions are $10 \%$ higher in the ED approximation, as expected from the results presented in Figure 1(b). The additional absorption peaks that can be assigned to electric-dipole-forbidden $1 s \rightarrow d_{x z}$ transitions are directly included in the BED spectrum. The intensities of the $1 s \rightarrow f$ transitions are orders of magnitude smaller than the $1 s \rightarrow p$ transitions, and they are thus not visible with the present choice of damping parameter $\gamma$.

\section{Trans-polyenes}

We now turn our attention to evaluate the significance of going beyond the ED approximation in spatially extended molecular systems. We consider a series of three progressively longer trans-polyenes (cf. Figure 6) for which the molecular

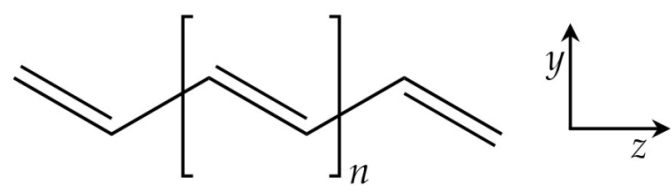

FIG. 6. Definition of the coordinate system used for the three studied polyenes; $n=8,13$, and 18 .
TABLE III. Comparison of imaginary and real components (a.u.) of the linear response functions [as defined in Eqs. (24) and (25)], and the underlying symmetry contributions, for the trans-polyenes of varying molecular length, i.e., the distance ( $\mathrm{nm}$ ) between the most distant $\mathrm{C}$-atoms, within and beyond the ED approximation computed at the CAM-B3LYP $100 \%$ /aug-cc-pVDZ level of theory at a resonant (off-resonant for the real part) X-ray photon energy $(\mathrm{eV})$. The corresponding wavelength $(\mathrm{nm})$ is given in parentheses. Propagation direction is along the $z$-axis and polarization direction along $x$ (cf. Figure 6).

\begin{tabular}{lccc}
\hline \hline & \multicolumn{3}{c}{ No. carbon atoms } \\
\cline { 2 - 4 } & 20 & 30 & 40 \\
\hline Length & 2.3 & 3.5 & 4.8 \\
$\hbar \omega$ & $274.3(4.52)$ & $274.3(4.52)$ & $274.3(4.52)$ \\
$-\operatorname{Im}\left\langle\left\langle\hat{p}_{x} ; \hat{p}_{x}\right\rangle\right\rangle_{\omega}$ & 1763.34 & 2735.16 & 3705.72 \\
$-\operatorname{Im}[G(-\omega ; \omega)]_{\mathrm{tot}}$ & 1762.99 & 2734.07 & 3703.85 \\
$-\operatorname{Im}[G(-\omega ; \omega)]_{\mathrm{A}_{\mathrm{u}}}$ & 988.145 & 1122.09 & 1918.46 \\
$-\operatorname{Im}[G(-\omega ; \omega)]_{\mathrm{Bg}}$ & 774.856 & 1611.98 & 1782.22 \\
$\hbar \omega$ & $217.7(5.70)$ & $217.7(5.70)$ & $217.7(5.70)$ \\
$-\operatorname{Re}\left\langle\left\langle\hat{p}_{x} ; \hat{p}_{x}\right\rangle\right\rangle_{\omega}$ & 21.4373 & 32.1935 & 42.9499 \\
$-\operatorname{Re}[G(-\omega ; \omega)]_{\mathrm{tot}}$ & 21.4418 & 32.2058 & 42.9685 \\
$-\operatorname{Re}[G(-\omega ; \omega)]_{\mathrm{A}_{\mathrm{u}}}$ & 13.6231 & 12.8981 & 18.5824 \\
$-\operatorname{Re}[G(-\omega ; \omega)]_{\mathrm{B}_{\mathrm{g}}}$ & 7.8187 & 19.3077 & 24.3861 \\
\hline \hline
\end{tabular}

length is comparable to the wavelength of radiation used in $\mathrm{X}$-ray spectroscopies addressing the $K$-edge.

In Table III, we report the imaginary part of the linear response function for the three polyenes within and beyond the ED approximation evaluated at the $1 s \rightarrow \pi^{*}$ band maximum. For the latter, we also provide the individual contributions from the excitations of $\mathrm{A}_{\mathrm{u}}$ and $\mathrm{B}_{\mathrm{g}}$ symmetries. Although the $\mathrm{BED}$ corrections increase with the length of the polyenes $(0.02 \%-0.05 \%$ across the series), the errors introduced by the ED approximation are negligible in the three cases considered. This observation is highly counterintuitive given the fact that the wavelength of radiation is $4.52 \mathrm{~nm}$ and the molecular length of the longest polyene $\left(\mathrm{C}_{40} \mathrm{H}_{42}\right)$ is $4.8 \mathrm{~nm}$. In this case, the situation is somewhat more complicated as compared to the atom because the transition density is distributed over equivalent carbon atoms that in many cases are separated by large distances. But here it is instead important to remember that the value of the reponse function is a summed total of contributions from each and every excited state in the system, and, in order to better understand how the response function can be rather insensitive to a BED treatment of the external fields, we must probe more individually the various absorption bands.

At a resonant frequency, the absorptive part of the response function provides information about the squared transition moment of the specific excitation (excitation manifold in this case) being probed. Accordingly, contributions from nonresonant transitions are strongly suppressed in the evaluation of response functions due to the much larger denominators in these cases. Therefore, to address the importance and sizes of BED corrections for other than $1 s \rightarrow \pi^{*}$ core excitations, we must alter our strategy. For this reason, we study in Table III also the real part of the response function, which is related to the refractive index, at a non-resonant, yet high frequency. 
In doing so, we focus on a property for which delocalized valence transitions can contribute more significantly, and, as a consequence, it could be anticipated that BED corrections would become important. Surprisingly, however, we find the BED corrections to the real part of the response function at the off-resonance frequency to be about as small as found for the imaginary part in the resonance region. Since the transition moments of at least the spatially extended valence excitations must be sensitive to field variations, the limited effect of the BED corrections must be due to their small contributions in the reponse function summation. In our quest to explain the made observations, we are led to examine the effect of BED on individual electronic transitions and study their contributions to the evaluation of the response function.

To get an idea about the extent to which field variations influence the individual contributions to the linear response function in a sum-over-states form [Eq. (23)], we inspect the norm of the transition moment for specific transitions at different frequencies [see Eq. (48)]. We focus on the shortest of the studied polyenes $\left(\mathrm{C}_{20} \mathrm{H}_{22}\right)$ with a molecular length of $2.3 \mathrm{~nm}$ and consider a selection of core excitations as well as the $\pi \rightarrow \pi^{*}$-valence excitation. The latter represents a delocalized excitation, where both the initial and final states extend over the entire molecular skeleton. The transition moments of core excitations are computed within the restricted-channel approximation, allowing excitations solely from the carbon core orbitals in order to describe the localized hole produced in the final state. Here, it becomes important to remember the symmetry of our system with inversion as one element, which means that, in the ED approximation, nonzero transition moments are limited to excited states of ungerade symmetry. In the BED realm, on the other hand, transitions of gerade symmetry become allowed and will enter the summation as response functions are evaluated.

We consider a setup with the electric field polarized along $x$ and propagating along the $z$-direction, see Figure 6 . In Figure 7, the frequency-dependent behavior of the norm of the transition moments for the lowest $1 s \rightarrow \pi^{*}$ excitations of $\mathrm{A}_{\mathrm{u}}$ and $\mathrm{B}_{\mathrm{g}}$ symmetry is depicted. It is a coincidential consequence due to the length of the chosen molecule that we see an almost perfect zero crossing for the transition moment of the $\mathrm{A}_{u}$ state at a photon energy corresponding to the core-valence excitation. The initial orbital involved in these two neardegenerate transitions is located on the two outermost carbons (one at each end). Included are also the values for the $\pi \rightarrow \pi^{*}$ valence excitation, where the polarization direction is along the $z$-axis and the propagation is along either the $x$ - or the $y$-axis. The transition moment norms obtained within the ED approximation are indicated by horizontal bars and excitation frequencies by vertical bars. Again, due to the presence of an inversion center, only the cosine term of the field-matter interaction operator contributes to the transition moments of the $A_{u}$ core and the valence excitations, whereas the sine term is active only for the $\mathrm{B}_{\mathrm{g}}$ core excitation. The BED corrections to the former $\left(\mathrm{A}_{\mathrm{u}}\right.$ symmetry) are therefore quadratic in $k$ at wavelengths large compared to the dimension of the transition, while linear for the $\mathrm{B}_{\mathrm{g}}$ excitation.

The BED corrections to the transition moments of the $\mathrm{A}_{\mathrm{u}}$ and $\mathrm{B}_{\mathrm{g}}$ core excitations, contributing to the $1 s \rightarrow \pi^{*}$ band,

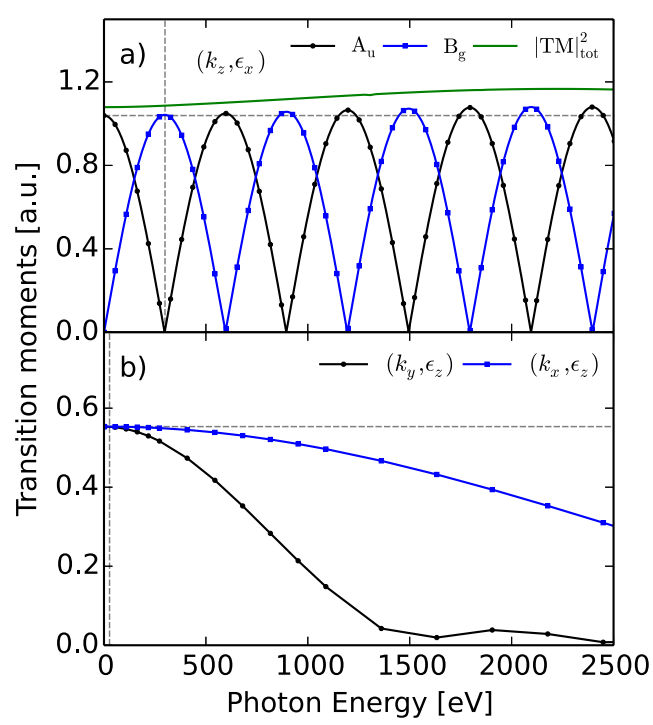

FIG. 7. BED transition moment norms [Eq. (48)], contributing to the refractive index at a given frequency, of (a) the lowest $\mathrm{A}_{\mathrm{u}}$ and $\mathrm{B}_{\mathrm{g}}$ transitions in the $1 s \rightarrow \pi^{*}$ band of $\mathrm{C}_{20} \mathrm{H}_{22}$, probed with an electromagnetic field polarized along $x$ and propagating along the long molecular axis, i.e., along the $z$-axis. The wavelengths of the oscillatory behavior relate to the long and short in-plane dimensions of the polyene. The green line is the sum of the norms of the transition moments of the $A_{u}$ and $B_{g}$ transitions. (b) The behavior of the $\pi \rightarrow \pi^{*}$ transition as probed with a $z$-polarized field, propagating along either the $x$ - or $y$-axis. Horizontal lines mark the non-vanishing ED transition moment norms (i.e., for transitions of ungerade symmetry), whereas vertical lines indicate the excitation energies.

oscillate with a frequency ( $\sim 44$ a.u.) that corresponds to a wavelength ( $\left.\sim 20 a_{0}\right)$ of about half the dimension of the long axis of the polyene (i.e., the distance between the origin and an outermost carbon). With reference to the discussion in Sec. V C, the spatial confinement of the $1 s$-orbital (estimated to be $r_{90 \%}=0.27$ a.u. for carbon in view of Figure 4) reduces the integration over the otherwise delocalized final $\pi^{*}$-orbital to a compact, atomic contribution from, in this case, the outermost carbons. Since the field variations over the extent of a single $1 s$-orbital is small, the atomic contribution will be well described within a local ED approximation, where the field strength has been scaled according to the distance between the two outermost atoms and the origin. However, the oscillating behavior of the individual excitation in the degenerate pair is removed when considering the sum of their transition moment norms, which represents the summed contribution to the linear response function from this degenerate pair of excited states. Indeed, the BED correction at the resonance frequency is negligible $(0.5 \%$ for the core excitation), as expected from the findings in Table III.

The $\pi \rightarrow \pi^{*}$ transition is significantly more sensitive to field variations, even across the short dimensions ( $x$ and $y$ ) of the molecule, as can be anticipated from its delocalized nature. The error in the squared transition moment introduced by the ED approximation at the frequency matching the core excitation, i.e., a contribution relevant for the real part of the response function ( $y$-propagation), amounts to $\sim 15 \%$ (compared to $0.1 \%$ at the resonance frequency). At higher frequencies, the transition moment approaches zero due to 
small contributions of opposite sign induced by the highfrequency oscillations across the dimension of the transition.

To summarize, it is clear that BED effects on the overall band profile in $K$-edge absorption spectra of the trans-polyene series are very small, but, when individual state resolution is reached, the picture becomes completely different and the effects are dramatic. While the underlying members of the degenerate $\left(A_{u}\right.$ and $\left.B_{g}\right)$ pairs oscillate strongly with the frequency, the cosine and sine behaviors are phase-shifted, and the sum of the transition moment norms, which enters the linear response function, is therefore only modestly affected. Furthermore, despite that the delocalized valence $\pi \rightarrow \pi^{*}$ transition is more sensitive to field variations than the core excitation, the magnitude of the BED corrections at the resonance frequency of the core excitation is too small with respect to the energy difference in the denominator to appreciably contribute to the refractive index at that frequency.

\section{CONCLUSION}

We have implemented the full semi-classical field-matter interaction operator in the complex polarization propagator formulation of response theory to describe molecular systems in the presence of weak, monochromatic, linearly polarized electromagnetic fields. Our formulation is inherently gaugeorigin independent. The implementation has been applied to study the significance of going beyond the electric-dipole approximation in three critical cases represented by (i) Xray absorption of high-energy photons, (ii) electric-dipoleforbidden transitions, and (iii) X-ray transitions in spatially extended systems. We illustrate these aspects by numerical results obtained for the alkaline earth metals as well as the series of trans-polyenes.

From the analysis of $K$-edge transitions in the alkaline earth metals, we find the $k r$ measure to nicely exhibit the expected linear dependence on the atomic number. This includes also $\mathrm{Ba}$ and $\mathrm{Ra}$, where relativistic effects have been neglected. Extrapolating the linear dependence to the maximum atomic number yields a $k r_{90 \%}$ value of $\sim 0.6$, which for pure $1 s \rightarrow n p$ transitions corresponds to an estimated maximum error of $20 \%$ upon restricting to the electric-dipole approximation. Thus, our results suggest that (i) for electricdipole-allowed transitions, the electric-dipole term remains the dominating contribution to the absorption cross section throughout the periodic table and (ii) a few-terms operator expansion captures the electric-dipole-forbidden transitions. In other words, the contraction of the $1 s$-orbital, which follows as the atomic number increases, is sufficiently strong (in terms of prefactor) to damp the effect of the concomitant increase in the photon energy across the periodic table.

The spatial extension of the final states of the $K$-edge $1 s \rightarrow \pi^{*}$ band in the series of trans-polyenes is comparable to the wavelength of the radiation field such that substantial field variations occur across the dimension of the transition. Indeed, for the individual excited states, the spatial field variations drastically influence the norm of the transition moments and any truncation of the exponential operator seems hopeless. However, when considering concurrently the degenerate electronic states, contributing to the $K$ edge band (i.e., summing over phase-shifted contributions), linear absorption is only modestly affected $(0.5 \%)$ by the infinite-order corrections, in line with the predictions based on the group 2 elements. This is a consequence of the localized nature of the initial state, which confines the pertinent transition density to the compact core hole. X-ray field variations become more pronounced when considering delocalized transition densities, such as valence $\pi \rightarrow \pi^{*}$ transitions; however, their impact on the physical absorption and refractive index is quenched by a large energy difference in the denominator of the sum-over-states property expressions.

\section{ACKNOWLEDGMENTS}

We acknowledge financial support from (N.H.L.) the Lundbeck Foundation, the Danish Council for Independent Research, (P.N.) Knut and Alice Wallenberg Foundation (Grant No. KAW-2013.0020), and the Swedish Research Council (Grant No. 621-2014-4646). Computational resources from the National Supercomputer Centre (NSC) in Sweden is acknowledged.

\section{APPENDIX A: GAUGE-ORIGIN INVARIANCE}

The individual transition moments, contained in the sumover-states expression in Eq. (23), depend on the gauge origin. However, the residues of the linear response function (i.e., norm of the transition moment) are gauge-origin invariant, as can be readily demonstrated by shifting the gauge origin according to $\boldsymbol{O}+\boldsymbol{a}$. Writing only the numerator explicitly yields

$$
\begin{aligned}
\langle 0| & \hat{V}^{-\omega}(\boldsymbol{O}+\boldsymbol{a})|n\rangle\left\langle n\left|\hat{V}^{\omega}(\boldsymbol{O}+\boldsymbol{a})\right| 0\right\rangle \\
& =\left\langle 0\left|e^{i \boldsymbol{k} \cdot(\boldsymbol{r}-\boldsymbol{a})}(\boldsymbol{\epsilon} \cdot \hat{\boldsymbol{p}})\right| n\right\rangle\left\langle n\left|e^{-i \boldsymbol{k} \cdot(\boldsymbol{r}-\boldsymbol{a})}(\boldsymbol{\epsilon} \cdot \hat{\boldsymbol{p}})\right| 0\right\rangle \\
& =e^{i \boldsymbol{k} \cdot(\boldsymbol{a}-\boldsymbol{a})}\left\langle 0\left|e^{i \boldsymbol{k r}}(\boldsymbol{\epsilon} \cdot \hat{\boldsymbol{p}})\right| n\right\rangle\left\langle n\left|e^{-i \boldsymbol{k} \cdot \boldsymbol{r}}(\boldsymbol{\epsilon} \cdot \hat{\boldsymbol{p}})\right| 0\right\rangle \\
& =\left\langle 0\left|\hat{V}^{-\omega}(\boldsymbol{O})\right| n\right\rangle\left\langle n\left|\hat{V}^{\omega}(\boldsymbol{O})\right| 0\right\rangle .
\end{aligned}
$$

Particularly, no exact-state conditions are invoked to prove the gauge-invariance of the non-expanded velocity form of the linear response function, and thus, it is equally valid in a finite basis.

\section{APPENDIX B: CONSTRUCTION OF PROPERTY INTEGRALS}

The Cartesian generalized transition moment integrals are evaluated following the McMurchie-Davidson scheme, ${ }^{67}$ in which the product of two primitive Cartesian Gaussians (the overlap distribution), needed for the evaluation of multiplicative one-electron operators, ${ }^{68}$ is expanded in Hermite Gaussian functions.

The Cartesian Gaussian functions are defined as

$$
\begin{aligned}
G_{i j k}(\boldsymbol{r}, a, \boldsymbol{A}) & =x_{A}^{i} y_{A}^{j} z_{A}^{k} e^{-a\left(x_{A}^{2}+y_{A}^{2}+z_{A}^{2}\right)} \\
& =G_{i}^{x}\left(x, a, A_{x}\right) G_{j}^{y}\left(y, a, A_{y}\right) G_{k}^{z}\left(z, a, A_{z}\right),
\end{aligned}
$$

where the favorable separability property into the Cartesian directions has been used in the second equality. The overlap 
distribution of Cartesian Gaussian functions can be written as a single Gaussian function centered at $\boldsymbol{P}$ between $\boldsymbol{A}$ and $\boldsymbol{B}$ and with exponent $p=a+b$. This allows us to expand the overlap distributions in Hermite Gaussian functions as

$$
\begin{aligned}
\Omega_{A B} & =G_{i j k}(\boldsymbol{r}, a, \boldsymbol{A}) G_{i^{\prime} j^{\prime} k^{\prime}}(\boldsymbol{r}, b, \boldsymbol{B}) \\
& =\sum_{t=0}^{i+i^{\prime}} \sum_{u=0}^{j+j^{\prime}} \sum_{v=0}^{k+k^{\prime}} E_{t}^{i i^{\prime}} E_{u}^{j j^{\prime}} E_{v}^{k k^{\prime}} \Lambda_{t u v}(\boldsymbol{r}, p, \boldsymbol{P}) .
\end{aligned}
$$

The Hermite Gaussians are defined as

$$
\Lambda_{t u v}(\boldsymbol{r}, p, \boldsymbol{P})=\left(\frac{\partial}{\partial P_{x}}\right)^{t}\left(\frac{\partial}{\partial P_{y}}\right)^{u}\left(\frac{\partial}{\partial P_{z}}\right)^{v} e^{-p r_{p}^{2}}
$$

and

$$
\boldsymbol{P}=\frac{a \boldsymbol{A}+b \boldsymbol{B}}{a+b}, \quad \boldsymbol{r}_{p}=\boldsymbol{r}-\boldsymbol{P}
$$

The expansion coefficients of the Hermite functions are evaluated recursively according to

$$
\begin{aligned}
E_{0}^{00} & =e^{-\frac{a b}{a+b}(\boldsymbol{A}-\boldsymbol{B})^{2}}, \\
E_{t}^{i+1, i^{\prime}} & =\frac{1}{2 p} E_{t-1}^{i i^{\prime}}+X_{P A} E_{t}^{i i^{\prime}}+(t+1) E_{t+1}^{i i^{\prime}}, \\
E_{t}^{i i^{\prime}+1} & =\frac{1}{2 p} E_{t-1}^{i i^{\prime}}+X_{P B} E_{t}^{i i^{\prime}}+(t+1) E_{t+1}^{i i^{\prime}},
\end{aligned}
$$

where $X_{P A}=P_{x}-A_{x}$. Similar expressions are obtained for the $y$ and $z$ components. Note that the expansion coefficients are independent of $\boldsymbol{r}$ and thus can be taken outside the integral over the electronic coordinates.

For the purpose of the contributions to the integrals over the $\hat{V}^{\omega}$ operator, which involves the linear momentum operator, we also define the differentiated overlap distribution,

$$
\begin{aligned}
\tilde{\Omega}_{i j}^{x} & =G_{i}^{x} \nabla_{x} G_{i^{\prime}}^{x}=\left(i^{\prime} G_{i}^{x} G_{i^{\prime}-1}^{x}-2 b G_{i}^{x} G_{i^{\prime}+1}^{x}\right) \\
& =\sum_{t=0}^{i+j}\left(E_{t}^{i i^{\prime}-1} i^{\prime}-2 b E_{t}^{i i^{\prime}+1}\right) \Lambda_{t}\left(x, p, P_{x}\right) \\
& =\sum_{t=0}^{i+j} \tilde{E}_{t}^{i i^{\prime}} \Lambda_{t}\left(x, p, P_{x}\right),
\end{aligned}
$$

which only differs from the overlap distribution in the expansion coefficients.

The $x, y$, and $z$ integral factors in the factorized form of the field-matter interaction operator integrals contain products of the (differentiated) overlap distribution and $\cos ^{m}\left(k_{\alpha} \alpha\right) \sin ^{1-m}\left(k_{\alpha} \alpha\right), m=0,1$. As exemplified by cosine, we have the following for the undifferentiated factors:

$$
\int_{-\infty}^{\infty} \Omega_{i i^{\prime}}^{x} \cos \left(k_{x} x\right) d x=\sum_{t=0}^{i+j} E_{t}^{i i^{\prime}} \nabla_{P_{x}}^{t} \int_{-\infty}^{\infty} e^{-p x_{p}^{2}} \cos \left(k_{x} x\right) d x .
$$

By using the cosine (sine) of sum identity (here for $x$ $\left.=x_{p}+P_{x}\right)$

$$
\begin{aligned}
& \cos (c \pm d)=\cos (c) \cos (d) \mp \sin (c) \sin (d), \\
& \sin (c \pm d)=\sin (c) \cos (d) \pm \cos (c) \sin (d),
\end{aligned}
$$

$$
\begin{aligned}
\int_{-\infty}^{\infty} e^{-p x_{p}^{2}} \cos \left(k_{x} x\right) d x_{p} \\
=\cos \left(k_{x} P_{x}\right) \int_{-\infty}^{\infty} e^{-p x_{p}^{2}} \cos \left(k_{x} x_{p}\right) d x_{p} \\
\quad-\sin \left(k_{x} P_{x}\right) \int_{-\infty}^{\infty} e^{-p x_{p}^{2}} \sin \left(k_{x} x_{p}\right) d x_{p} \\
=\cos \left(k_{x} P_{x}\right)\left(\frac{\pi}{p}\right)^{\frac{1}{2}} e^{-k_{x}^{2} / 4 p},
\end{aligned}
$$

where the sine integral vanishes due to the integrand being an odd function of $x_{p}$. Substituting this into Eq. (B9) yields

$$
\int_{-\infty}^{\infty} \Omega_{i i^{\prime}}^{x} \cos \left(k_{x} x\right) d x=\left(\frac{\pi}{p}\right)^{\frac{1}{2}} e^{-k_{x}^{2} / 4 p} \sum_{t=0}^{i+j} E_{t}^{i i^{\prime}} \nabla_{P_{x}}^{t} \cos \left(k_{x} P_{x}\right),
$$

where the $n$th order derivatives of cosine and sine are given as

$$
\nabla_{x}^{n} \cos (a x+b)=\left\{\begin{array}{cl}
(-1)^{k} a^{n} \cos (a x+b), & n=2 k \\
(-1)^{k+1} a^{n} \sin (a x+b), & n=2 k+1
\end{array}\right.
$$

and

$$
\nabla_{x}^{n} \sin (a x+b)=\left\{\begin{array}{ll}
(-1)^{k} a^{n} \sin (a x+b), & n=2 k \\
(-1)^{k} a^{n} \cos (a x+b), & n=2 k+1
\end{array},\right.
$$

respectively. The differentiated factors can now be determined by replacing the overlap distribution with the differentiated version to give

$$
\begin{aligned}
\int_{-\infty}^{\infty} \tilde{\Omega}_{i i^{\prime}}^{x} \cos \left(k_{x} x\right) d x= & \left(\frac{\pi}{p}\right)^{\frac{1}{2}} e^{-k_{x}^{2} / 4 p} \\
& \times \sum_{t=0}^{i+j} \tilde{E}_{t}^{i i^{\prime}} \nabla_{P_{x}}^{t} \cos \left(k_{x} P_{x}\right)
\end{aligned}
$$

With the (differentiated) overlap distributions at hand, Eq. (B16) gives a recipe for the evaluation of the Cartesian factors, contributing to a given integral of the field-matter interaction operator.

The field-matter interaction operator mixes all symmetries. Therefore, to exploit the Abelian symmetry handling available in the DALTON program, the 24 real and imaginary contributions, obtained by combining each term in Eqs. (44) and (45), respectively, with the three components of the linear momentum operator, are collected according to irreducible representation and treated separately. The contributions to the linear response function from the irreducible representations are re-combined upon evaluation of the total linear response function. 
${ }^{5} \mathrm{P}$. Norman, "A perspective on nonresonant and resonant electronic response theory for time-dependent molecular properties," Phys. Chem. Chem. Phys. 13, 20519-20535 (2011).

${ }^{6} \mathrm{~K}$. Schwarzschild, "Zur elektrodynamik. I. Zwei formen des princips der action in der elektronentheorie," Gött. Nachr., Math.-Phys. K1. 1903, 126-131.

${ }^{7}$ M. Gell-Mann, "The interpretation of the new particles as displaced charge multiplets," Il Nuovo Cimento 4, 848-866 (1956).

${ }^{8}$ S. D. George, T. Petrenko, and F. Neese, "Time-dependent density functional calculations of ligand K-edge X-ray absorption spectra," Inorg. Chim. Acta 361, 965-972 (2008)

${ }^{9}$ N. Lee, T. Petrenko, U. Bergmann, F. Neese, and S. DeBeer, "Probing valence orbital composition with iron $\mathrm{K}_{\beta} \mathrm{X}$-ray emission spectroscopy," J. Am. Chem. Soc. 132, 9715-9727 (2010).

${ }^{10} \mathrm{~S}$. Bernadotte, A. J. Atkins, and C. R. Jacob, "Origin-independent calculation of quadrupole intensities in X-ray spectroscopy," J. Chem. Phys. 137, 204106 (2012)

${ }^{11}$ D. P. Craig and T. Thirunamachandran, Molecular Quantum Electrodynamics: An Introduction to Radiation-Molecule Interactions (Courier Dover Publications, 1998).

${ }^{12}$ D. W. Lindle and O. Hemmers, "Breakdown of the dipole approximation in soft-X-ray photoemission," J. Electron Spectrosc. Relat. Phenom. 100, 297-311 (1999).

${ }^{13}$ O. Hemmers, R. Guillemin, and D. W. Lindle, "Nondipole effects in soft X-ray photoemission,” Radiat. Phys. Chem. 70, 123-147 (2004).

${ }^{14}$ P. V. Demekhin, "On the breakdown of the electric dipole approximation for hard X-ray photoionization cross sections," J. Phys. B: At., Mol. Opt. Phys. 47, 025602 (2014).

${ }^{15}$ G. M. Seabra, I. G. Kaplan, and J. V. Ortiz, "Molecular photoionization cross sections in electron propagator theory: Angular distributions beyond the dipole approximation," J. Chem. Phys. 123, 114105 (2005).

${ }^{16} \mathrm{~J}$. Stöhr, in NEXAFS Spectroscopy (Springer, 1992), Vol. 25.

${ }^{17}$ T. Glaser, B. Hedman, K. O. Hodgson, and E. I. Solomon, "Ligand K-edge X-ray absorption spectroscopy: A direct probe of ligand-metal covalency," Acc. Chem. Res. 33, 859-868 (2000), pMID: 11123885.

${ }^{18}$ E. I. Solomon, B. Hedman, K. O. Hodgson, A. Dey, and R. K. Szilagyi, "Ligand K-edge X-ray absorption spectroscopy: Covalency of ligand-metal bonds," Coord. Chem. Rev. 249, 97-129 (2005).

${ }^{19}$ F. Neese, B. Hedman, K. O. Hodgson, and E. I. Solomon, "Relationship between the dipole strength of ligand pre-edge transitions and metal-ligand covalency," Inorg. Chem. 38, 4854-4860 (1999).

${ }^{20}$ L. S. Kau, D. J. Spira-Solomon, J. E. Penner-Hahn, K. O. Hodgson, and E. I. Solomon, "X-ray absorption edge determination of the oxidation state and coordination number of copper. Application to the type 3 site in rhus vernicifera laccase and its reaction with oxygen," J. Am. Chem. Soc. 109, 6433-6442 (1987).

${ }^{21}$ F. W. Lytle, R. B. Greegor, and A. J. Panson, "Discussion of X-rayabsorption near-edge structure: Application to $\mathrm{Cu}$ in the high- $T_{c}$ superconductors $\mathrm{La}_{1.8} \mathrm{Sr}_{0.2} \mathrm{CuO}_{4}$ and $\mathrm{YBa}_{2} \mathrm{Cu}_{3} \mathrm{O}_{7}$," Phys. Rev. B 37, 1550-1562 (1988).

${ }^{22}$ E. M. C. Alayon, M. Nachtegaal, E. Kleymenov, and J. A. van Bokhoven, "Determination of the electronic and geometric structure of $\mathrm{Cu}$ sites during methane conversion over Cu-MOR with X-ray absorption spectroscopy," Microporous Mesoporous Mater. 166, 131-136 (2013).

${ }^{23}$ T. Ressler, J. Wienold, R. E. Jentoft, and T. Neisius, "Bulk structural investigation of the reduction of $\mathrm{MoO}_{3}$ with propene and the oxidation of $\mathrm{MoO}_{2}$ with oxygen," J. Catal. 210, 67-83 (2002).

${ }^{24}$ J. J. Rehr and R. C. Albers, "Theoretical approaches to X-ray absorption fine structure,” Rev. Mod. Phys. 72, 621-654 (2000).

${ }^{25}$ V. K. Yachandra, "X-ray absorption spectroscopy and applications in structural biology," Methods Enzymol. 246, 638-675 (1995).

${ }^{26}$ R. K. Hocking, S. Debeer George, Z. Gross, F. A. Walker, K. O. Hodgson, B. Hedman, and E. I. Solomon, "Fe L-and K-edge XAS of low-spin ferric corrole: Bonding and reactivity relative to low-spin ferric porphyrin," Inorg. Chem. 48, 1678-1688 (2009)

${ }^{27}$ S. DeBeer George, T. Petrenko, and F. Neese, "Prediction of iron K-edge absorption spectra using time-dependent density functional theory," J. Phys. Chem. A 112, 12936-12943 (2008).

${ }^{28} \mathrm{G}$. Hähner, "Near edge X-ray absorption fine structure spectroscopy as a tool to probe electronic and structural properties of thin organic films and liquids," Chem. Soc. Rev. 35, 1244-1255 (2006).

${ }^{29}$ F. Bournel, C. Laffon, P. Parent, and G. Tourillon, "Adsorption of some substituted ethylene molecules on Pt (111) at $95 \mathrm{~K}$ part 1: NEXAFS, XPS and UPS studies," Surf. Sci. 350, 60-78 (1996).
${ }^{30}$ C. Vahlberg, M. Linares, S. Villaume, P. Norman, and K. Uvdal, "Noradrenaline and a thiol analogue on gold surfaces: An infrared reflectionabsorption spectroscopy, X-ray photoelectron spectroscopy, and near-edge X-ray absorption fine structure spectroscopy study,” J. Phys. Chem. C 115, 165-175 (2011)

${ }^{31}$ C. Vahlberg, M. Linares, P. Norman, and K. Uvdal, "Phenylboronic esterand phenylboronic acid-terminated alkanethiols on gold surfaces," J. Phys. Chem. C 116, 796-806 (2012).

${ }^{32}$ R. Vedrinskii, V. Kraizman, A. Novakovich, P. V. Demekhin, S. Urazhdin, B. Ravel, and E. Stern, "Pre-edge fine structure (PEFS) of the K-XAS for the $3 \mathrm{~d}$ atoms in compounds: A new tool for quantitative atomic structure determination," J. Phys. IV 7, C2-107 (1997).

${ }^{33}$ R. V. Vedrinskii, V. L. Kraizman, A. A. Novakovich, P. V. Demekhin, and S. V. Urazhdin, "Pre-edge fine structure of the $3 \mathrm{~d}$ atom K X-ray absorption spectra and quantitative atomic structure determinations for ferroelectric perovskite structure crystals," J. Phys.: Condens. Matter 10, 9561 (1998).

${ }^{34}$ B. M. Messer, C. D. Cappa, J. D. Smith, W. S. Drisdell, C. P. Schwartz, R. C. Cohen, and R. J. Saykally, "Local hydration environments of amino acids and dipeptides studied by X-ray spectroscopy of liquid microjets," J. Phys. Chem. B 109, 21640-21646 (2005).

${ }^{35}$ B. M. Messer, C. D. Cappa, J. D. Smith, K. R. Wilson, M. K. Gilles, R. C. Cohen, and R. J. Saykally, "pH dependence of the electronic structure of glycine," J. Phys. Chem. B 109, 5375-5382 (2005).

${ }^{36}$ H. Mahr, "Two-photon absorption spectroscopy," in Quantum Electronics Nonlinear Optics, Part A, edited by H. Rabin and C. L. Tang (Academic Press, New York, 1975), Vol. 1.

${ }^{37}$ P. Norman, D. M. Bishop, H. J. Aa. Jensen, and J. Oddershede, "Nearresonant absorption in the time-dependent self-consistent field and multiconfigurational self-consistent field approximations,' J. Chem. Phys. 115, 10323 (2001).

${ }^{38}$ P. Norman, D. M. Bishop, H. J. Aa. Jensen, and J. Oddershede, "Nonlinear response theory with relaxation: The first-order hyperpolarizability," J. Chem. Phys. 123, 194103 (2005).

${ }^{39}$ U. Ekström and P. Norman, "X-ray absorption spectra from the resonantconvergent first-order polarization propagator approach," Phys. Rev. A 74, 042722 (2006).

${ }^{40}$ G. C. Schatz and M. A. Ratner, Quantum Mechanics in Chemistry (Courier Corporation, 2002).

${ }^{41}$ T. Helgaker, S. Coriani, P. Jørgensen, K. Kristensen, J. Olsen, and K. Ruud, "Recent advances in wave function-based methods of molecular-property calculations," Chem. Rev. 112, 543-631 (2012).

${ }^{42}$ D. J. Griffiths, Introduction to Electrodynamics (Pearson, 2013).

${ }^{43}$ J. Olsen and P. Jørgensen, "Linear and non-linear response functions for an exact state and for an MCSCF state,' J. Chem. Phys. 82, 3235-3264 (1985).

${ }^{44}$ V. M. Agranovich and V. L. Ginzburg, Spatial Dispersion in Crystal Optics and the Theory of Excitons (Interscience London, 1966).

${ }^{45}$ F. Bechstedt, Many-Body Approach to Electronic Excitations (Springer, 2014).

${ }^{46}$ A. S. Davydov, Quantum Mechanics, 2nd ed. (Pergamon, Oxford, 1976).

${ }^{47}$ R. Bast, J. Juselius, and T. Saue, "4-component relativistic calculation of the magnetically induced current density in the group 15 heteroaromatic compounds," Chem. Phys. 356, 187-194 (2009).

${ }^{48}$ E. Merzbacher, Quantum Mechanics (Wiley, 1970).

${ }^{49}$ B. H. Bransden and C. J. Joachain, Physics of Atoms and Molecules, 2 nd ed. (Pearson Education India, 2003).

${ }^{50}$ K. Aidas, C. Angeli, K. L. Bak, V. Bakken, R. Bast, L. Boman, O. Christiansen, R. Cimiraglia, S. Coriani, P. Dahle, E. K. Dalskov, U. Ekström, T. Enevoldsen, J. J. Eriksen, P. Ettenhuber, B. Fernández, L. Ferrighi, H. Fliegl, L. Frediani, K. Hald, A. Halkier, C. Hättig, H. Heiberg, T. Helgaker, A. C. Hennum, H. Hettema, E. Hjertenæs, S. Høst, I.-M. Høyvik, M. F. Iozzi, B. Jansik, H. J. A. Jensen, D. Jonsson, P. Jørgensen, J. Kauczor, S. Kirpekar, T. Kjærgaard, W. Klopper, S. Knecht, R. Kobayashi, H. Koch, J. Kongsted, A. Krapp, K. Kristensen, A. Ligabue, O. B. Lutnæs, J. I. Melo, K. V. Mikkelsen, R. H. Myhre, C. Neiss, C. B. Nielsen, P. Norman, J. Olsen, J. M. H. Olsen, A. Osted, M. J. Packer, F. Pawlowski, T. B. Pedersen, P. F. Provasi, S. Reine, Z. Rinkevicius, T. A. Ruden, K. Ruud, V. Rybkin, P. Salek, C. C. M. Samson, A. S. de Merás, T. Saue, S. P. A. Sauer, B. Schimmelpfennig, K. Sneskov, A. H. Steindal, K. O. Sylvester-Hvid, P. R. Taylor, A. M. Teale, E. I. Tellgren, D. P. Tew, A. J. Thorvaldsen, L. Thøgersen, O. Vahtras, M. A. Watson, D. J. D. Wilson, M. Ziolkowski, and H. Ågren, "The Dalton quantum chemistry program system,” Wiley Interdiscip. Rev.: Comput. Mol. Sci. 4, 269 (2014).

${ }^{51}$ Dalton, A Molecular Electronic Structure Program, Release DALTON2013.0, 2013, see http://daltonprogram.org/. 
${ }^{52}$ J. Kauczor, P. Jørgensen, and P. Norman, "On the efficiency of algorithms for solving Hartree-Fock and Kohn-Sham response equations," J. Chem. Theory Comput. 7, 1610-1630 (2011).

${ }^{53} \mathrm{~J}$. Kauczor and P. Norman, "Efficient calculations of molecular linear response properties for spectral regions," J. Chem. Theory Comput. 10, 2449-2455 (2014).

${ }^{54}$ P. Sałek, O. Vahtras, T. Helgaker, and H. Ågren, "Density-functional theory of linear and nonlinear time-dependent molecular properties," J. Chem. Phys. 117, 9630-9645 (2002).

${ }^{55} \mathrm{O}$. Christiansen, P. Jørgensen, and C. Hättig, "Response functions from Fourier component variational perturbation theory applied to a timeaveraged quasienergy," Int. J. Quantum Chem. 68, 1-52 (1998).

${ }^{56}$ M. J. Frisch, G. W. Trucks, H. B. Schlegel, G. E. Scuseria, M. A. Robb, J. R. Cheeseman, G. Scalmani, V. Barone, B. Mennucci, G. A. Petersson, H. Nakatsuji, M. Caricato, X. Li, H. P. Hratchian, A. F. Izmaylov, J. Bloino, G. Zheng, J. L. Sonnenberg, M. Hada, M. Ehara, K. Toyota, R. Fukuda, J. Hasegawa, M. Ishida, T. Nakajima, Y. Honda, O. Kitao, H. Nakai, T. Vreven, J. A. Montgomery, Jr., J. E. Peralta, F. Ogliaro, M. Bearpark, J. J. Heyd, E. Brothers, K. N. Kudin, V. N. Staroverov, R. Kobayashi, J. Normand, K. Raghavachari, A. Rendell, J. C. Burant, S. S. Iyengar, J. Tomasi, M. Cossi, N. Rega, J. M. Millam, M. Klene, J. E. Knox, J. B. Cross, V. Bakken, C. Adamo, J. Jaramillo, R. Gomperts, R. E. Stratmann, O. Yazyev, A. J. Austin, R. Cammi, C. Pomelli, J. W. Ochterski, R. L. Martin, K. Morokuma, V. G. Zakrzewski, G. A. Voth, P. Salvador, J. J. Dannenberg, S. Dapprich, A. D. Daniels, Ö. Farkas, J. B. Foresman, J. V. Ortiz, J. Cioslowski, and D. J. Fox, Gaussian 09 Revision D.01, Gaussian Inc., Wallingford, CT, 2009.

${ }^{57}$ P. J. Stephens, F. J. Devlin, C. F. Chabalowski, and M. J. Frisch, "Ab initio calculation ofvibrational absorption and circular dichroism spectra using density functional force fields," J. Phys. Chem. 98, 11623-11627 (1994).

${ }^{58}$ A. D. Becke, "Density-functional thermochemistry. III. The role of exact exchange," J. Chem. Phys. 98, 5648-5652 (1993).

${ }^{59}$ S. H. Vosko, L. Wilk, and M. Nusair, "Accurate spin-dependent electron liquid correlation energies for local spin density calculations: A critical analysis," Can. J. Phys. 58, 1200-1211 (1980).
${ }^{60}$ C. Lee, W. Yang, and R. G. Parr, "Development of the Colle-Salvetti correlation-energy formula into a functional of the electron density," Phys. Rev. B 37, 785-789 (1988).

${ }^{61}$ T. H. Dunning, "Gaussian basis sets for use in correlated molecular calculations. I. The atoms boron through neon and hydrogen," J. Chem. Phys. 90, 1007-1023 (1989).

${ }^{62}$ T. Yanai, D. P. Tew, and N. C. Handy, "A new hybrid exchange-correlation functional using the coulomb-attenuating method (CAM-B3LYP)," Chem. Phys. Lett. 393, 51-57 (2004).

${ }^{63}$ D. E. Woon and T. H. Dunning, Jr., "Gaussian basis sets for use in correlated molecular calculations. IV. Calculation of static electrical response properties," J. Chem. Phys. 100, 2975-2988 (1994).

${ }^{64}$ B. O. Roos, V. Veryazov, and P.-O. Widmark, "Relativistic atomic natural orbital type basis sets for the alkaline and alkaline-earth atoms applied to the ground-state potentials for the corresponding dimers," Theor. Chim. Acta 111, 345-351 (2004).

${ }^{65}$ A. Kramida, Yu. Ralchenko, J. Reader, and NIST ASD Team, NIST Atomic Spectra Database, version 5.2 (National Institute of Standards and Technology, Gaithersburg, MD, 2014), available online at http://physics.nist.gov/ asd.

${ }^{66}$ C. S. Wood, S. C. Bennett, D. Cho, B. P. Masterson, J. L. Roberts, C. E. Tanner, and C. E. Wieman, "Measurement of parity nonconservation and an anapole moment in cesium," Science 275, 1759-1763 (1997).

${ }^{67}$ L. E. McMurchie and E. R. Davidson, "One- and two-electron integrals over Cartesian Gaussian functions," J. Comput. Phys. 26, 218-231 (1978).

${ }^{68}$ T. Helgaker, P. Jørgensen, and J. Olsen, Molecular Electronic-Structure Theory (Wiley, 2000).

${ }^{69}$ K. G. Dyall, "Relativistic double-zeta, triple-zeta, and quadruple-zeta basis sets for the 4s, 5s, 6s, and 7s elements," J. Phys. Chem. A 113, 12638-12644 (2009).

${ }^{70} \mathrm{~L}$. Visscher, "Approximate molecular relativistic Dirac-Coulomb calculations using a simple Coulombic correction," Theor. Chem. Acc. 98, 68-70 (1997).

${ }^{71}$ J. A. Bearden and A. Burr, "Reevaluation of X-ray atomic energy levels," Rev. Mod. Phys. 39, 125-142 (1967). 